\title{
Research Paper \\ Identifying Barriers to Self-advocacy in Women With Multiple Sclerosis in Iran: A Qualitative Study
}

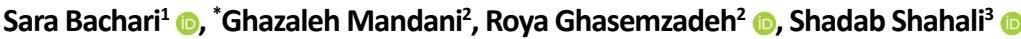

1. Department of Rehabilitation Administration, School of Rehabilitation, Ahvaz Jundishapur University of Medical Sciences, Ahvaz, Iran.

2. Department of Occupational Therapy, Musculoskeletal Rehabilitation Research Center, School of Rehabilitation, Ahvaz Jundishapur University of Medical Science, Ahvaz, Iran 3. Department of Reproductive Health and Midwifery, School of Medical Sciences, Tarbiat Modares University, Tehran, Iran.

\begin{tabular}{|c|c|}
\hline $\begin{array}{l}\text { Use your device to scan } \\
\text { and read the article online }\end{array}$ & \\
\hline 口ising & $\begin{array}{l}\text { dration Bachari S, Mandani Gh, Ghasemzadeh R, Shahali Sh. [Identifying Barriers to Self-advocacy in Women With Mul- } \\
\text { tiple Sclerosis in Iran: A Qualitative Study (Persian)]. Archives of Rehabilitation. 2021; 22(3):378-393. https://doi.org/10.32598/ } \\
\text { RJ.22.3.3277.1 }\end{array}$ \\
\hline aring & dol'https://doi.org/10.32598/RJ.22.3.3277.1 \\
\hline
\end{tabular}

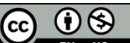

Received: 04 Jan 2021 Accepted: 15 May 2021 Available Online: 01 Oct 2021

Keywords: Multiple Sclerosis, Self-advocacy, Women, Qualitative content analysis

\section{A B STRACT}

Objective Chronic Multiple Sclerosis (MS) often occurs in middle-aged people, who are supposed to take their most important roles in the family and community and thus affect their self-advocacy. This study aimed to identify the barriers to self-advocacy in women with MS.

Materials \& Methods This study was conducted using a qualitative content analysis method. The participants were 10 female members of the MS Association of Khuzestan Province of Iran aged 30-40 years with different educational levels, marital status, and occupations. They were selected using a purposive sampling method. The study data were collected through semi-structured interviews and continued until data saturation from June to November 2019. The content analysis was performed using Lundman and Graneheim's qualitative content analysis method with an inductive approach conducted continuously and simultaneously with data collection. To evaluate the validity and reliability of qualitative data, we used Lincoln and Guba criteria (credibility, dependability, conformability, and transferability). Results The data analysis revealed 900 codes, 21 subcategories, and 6 themes of family barriers, medical barriers, job-related barriers, environmental barriers, sociocultural barriers, and psychological barriers. Conclusion Women with MS face a variety of barriers to self-advocacy; most of them are related to family, environment, and society, in addition to personal barriers. Social support and the availability of sufficient and appropriate information about MS can play an important role in confronting these women with mentioned barriers and realizing their self-advocacy.

\footnotetext{
English Version M

Introduction

ultiple Sclerosis (MS) is an autoimmune, debilitating, and chronic disease whose etiology is unknown. MS has neither a preventive measure nor a definitive cure. The goal of treat-

ment is to manage symptoms, maintain function, and slow the progression of the disease [1-3]. About $75 \%$ of people with MS are women, and the disease is mainly seen in middle-aged women (around 30 years old) [1]. Women with disabilities in Iran face discrimination due to their physical disabilities and gender and are ignored in many sections of society $[1,4]$. Although people may sometimes need support, they are entitled to take control of the existing condi-

\section{"Corresponding Author:}

Ghazale Mandani, PhD.

Address: Department of Occupational Therapy, Musculoskeletal Rehabilitation Research Center, School of Rehabilitation, Ahvaz Jundishapur University of Medical Science, Ahvaz, Iran.

Tel: +98 (912) 4868629

E-Mail: ghazal.mandani@gmail.com
} 
tions and resources $[5,6]$. This state is called self-advocacy, which means that people with disabilities can speak recklessly, express their desires, and be their own voice $[5,6]$. Identifying barriers to self-advocacy and overcoming them in women with MS requires knowing those barriers in women's context and culture. People's self-advocacy experience is an entirely subjective phenomenon, and qualitative research can study it. Studies conducted in Iran on MS have mostly examined the epidemiological and quantitative aspects of the disease in patients and caregivers, and qualitative studies on MS are a few [7-9]. We found no investigation in Iran on the self-advocacy of MS patients. In studies conducted in other countries, the concept of self-advocacy has been studied in relation to other concepts or focused on teaching self-advocacy skills. In this regard, this study aims to investigate the barriers to self-advocacy in women with MS in Iran using a qualitative approach.

\section{Materials and Methods}

This research is a qualitative study using conventional content analysis. This method is usually appropriate when existing theories or literature on the research topic are limited. In this method, codes are extracted directly or indirectly from the data [10]. The study population was the members of the MS Association of Khuzestan Province, Iran. The samples were 10 women with MS for at least 1 year. The selection of samples was made using a purposive sampling method by taking into account the maximum diversity in terms of age, educational level, marital status, family, social conditions, and so on. The sampling was continued until the data saturation. The inclusion criteria were being diagnosed with MS or exposure to this disease. The exclusion criteria were the inability to participate in the study, unwillingness to talk about personal and family issues, reluctance to continue participation, and inability to perceive the questions.

Data were collected during in-depth semi-structured interviews with the patients for 60-90 minutes. The interview began with open-ended questions, followed by exploratory questions. All interviews were recorded with the participants' permission, and field notes were taken to supplement the findings. The interviews continued until data saturation that was obtained after seven interviews, and the further three interviews were performed to ensure saturation. In this regard, the interview was conducted in two sessions for one of the participants. The research environment was determined according to their conditions and comfort.

To analyze the data, we used the qualitative content analysis using the Lundman and Graneheim method [11]. In this regard, the interviews were transcribed, and the investigation began after reading them. Then, the key concepts (themes) were highlighted, and the important parts were extracted and sorted into the smallest units (codes). A table of three columns was then designed, and a list of themes was prepared; after reviewing and finding similarities in meaning and concept, the subcategories were placed in each related column.

To evaluate the validity and reliability of qualitative data, we used the Lincoln and Guba criteria (credibility, dependability, conformability, and transferability) [8]. The credibility of the data was achieved through the researcher's constant engagement with the data and their comparison, as well as the opinions of two experts (not a part of the research team). Dependability of the data was achieved using the opinions of participants and some colleagues about the accuracy of data analysis. By submitting reports to 4 professors and their approval, the conformability of the data was confirmed. The samples were selected, taking into account the maximum diversity. To achieve transferability, the researcher took notes and recorded all ideas and activities on collecting data and analyzing them over time so that other people could follow up on the issue by reading these notes or continuing this research.

\section{Results}

The participants were employed women or homemakers with MS in the third or fourth decades of their lives, with different marital statuses and educational levels. As a result of analyzing, 900 codes, 21 subcategories, and 6 themes were obtained.

\section{Family-related barriers}

This theme had four subcategories of "not supported by the husband", "parents' fear of their child's future", "nonacceptance by the family", and "family and husband interference in the pursuit of rights". Findings showed that the occurrence of chronic diseases such as MS puts the patients in a situation where they feel the need to be supported by the family and others. Some participants believed that the family's neglect and inattention to them and their wishes had worsened their illnesses. Some women, on the other hand, faced challenges such as distancing from their husbands, husbands' absence in urgent circumstances and leaving them: "... My husband was in shock after my illness. After a while, he formally asked for a divorce” (participant No. 2).

\section{Job-Related barriers}

This theme had three subcategories of "lack of job security", "ineffective objections", and "pressures from the workplace". Employees often spend considerable time at the workplace, and their current situation can play a sig- 
nificant role in their experiences of MS. Lack of job security was one of the most frequently mentioned barriers. For this reason, some of them refused to express their disease and consequently could not defend their own rights as they should according to their physical and psychological conditions. Others argued that expressing their disease and selfadvocacy would lead to job losses because most employers might refuse to hire them. Some believed that even if they were trying to advocate their position, their efforts would still be ignored.

\section{Medical barriers}

The subcategories of this theme are "medical shortcomings", "poor performance of medical staff", "lack of privacy in medical centers", "limitations related to medications", and "poor economic status". In some participants, the physician's inappropriate behavior when making the diagnosis had shocked them and created an intense fear and distrust of the physician. Moreover, according to some patients, not providing details and descriptions of the disease conditions can have a significant impact on the realization of their selfadvocacy and can be challenging: "Unfortunately, the doctors have to be forced to get information!" (Participant No. 1). Lack of a private space can also be a barrier for the patient to express their expectations better and demands of the physician and treatment staff: "I wanted to ask a question, but the next patient [suddenly] came inside and then I felt shy to ask anything [related to the disease]". (Participant No. 6). Any problem with the preparation, use, and even effects of medications can lead to a variety of physical and mental experiences for people with MS: "The days I take cinnoVex injections, I feel a lot of pain in my body; the condition of someone who has a cold: headache, body aches, bruises" (Participant No. 8). The problems with medication supply, accessibility of medications, and receiving and injecting specialty drugs were other issues that the participants reported, especially those who live far from the province's capital. Economic issues and problems were other limitations that participants had experienced.

\section{Environmental barriers}

This theme included two subcategories of "environmental barriers to education" and "access and geographical limitations". The place of education and training is one of the important environments in which people attend and take on various roles. Lack of proper cooperation and empathy in these environments can lead to inadequate self-advocacy in participants. One of the participants, who had difficulty attending university and taking exams due to her medical conditions, did not receive the necessary support to compensate for this absence, despite the follow-up and persis- tence of her professor: “... I texted them every day for two weeks to ask what is the alternative way, but they did not answer me at all, until one day before the exam when they told me to take the exam online with others" (Participant No. 2). Several women had restrictions and lack of access to appropriate medical facilities: "The Association is far away [from my house], so I cannot communicate with them very much ..". (Participant No. 1). Some reported the concentration of medical facilities in the provincial and capital centers: "None of the cities in Iran has the facilities that exist in Tehran" (Participant No. 4).

\section{Sociocultural barriers}

This theme includes two subcategories of "stigma and judgment" and "social barriers". Fear of being labeled, judged, or seen as weak were the feelings reported by the majority of women. In many cases, this caused the person to be silent or isolated in situations that required her to speak. Participants often believed that because of the negative view in society, judgments lead to pity in others and inadvertently instill in them a sense of disability.

\section{Psychological barriers}

This theme included five subcategories of "distrust and pessimism about the health care system, family, and society", "ignoring of the rights", "feeling of inadequacy", "fear of the future", and "psychological consequences of MS". Distrust and pessimism were the significant psychological barriers that women experienced in different situations, which usually led to the feeling of the incapability in them. Family's lack of support for the expression of needs and not being optimistic about the outcome of their expression were other barriers to the realization of self-advocacy in women and caused them not to pursue and insist on their needs. The feeling of inadequacy in personal life was another problem for participants, which was experienced due to the diminution of their roles as a wife, mother, or child and following the consequences of their disease. This condition reduced their ability to advocate. The challenges posed by these issues and the uncertainty about the process of MS can lead to various fears for the patient. Fear of the uncertain future, fear of facing peers, and even worries about the future and health of children were other barriers in women.

\section{Discussion and Conclusion}

The present study tried to provide a ground for improving the quality of life and realizing the rights of MS women by strengthening and emphasizing their self-advocacy through finding its barriers. The six obstacles encountered in this study are very similar to the findings of Kaldi and Salah- 
shouri [12]. They believed that women's empowerment and strengthening of their self-advocacy depends on many internal and external factors, such as the physical and mental statuses of women and their family income level. The six identified barriers can be examined from other aspects in two categories of environmental (external) and individual (internal) because the nature of MS is such that it threatens the independence and ability of self-care in patients to play an effective role in the family and society and often creates a sense of uncertainty in them [7].

Family-related barriers as one of the identified barriers indicate that the family's refusal to accept the disease in women can create limitations. Although the results of Sadat et al. showed the great effect of MS on family members, which is consistent with our results, they stated that these effects in some cases over time lead to family's misbehavior, causing discouragement of the patient from the treatment of MS and or rejecting therapy [13]. This finding is inconsistent with our results; women in our study not only did not face family misbehavior and rejection over time but also their relationships in some cases improved and received significant attention from their family (due to fear and worry of parents about the future of their children). In some cases, the excessive attention of their husbands affected the independence of women to advocate themselves.

Job-related barriers were another finding of this study. Simmons et al. showed that many people with MS have difficulty keeping their jobs, even during good economic times [14]. Moore et al. also pointed to the job challenges of MS patients [15]. Dyck and Jongbloed showed that nonmedical factors, such as modifying work conditions and understanding employers with the possibility of delegating household tasks, can improve MS women's ability to work [16]. These findings are consistent with our results. What hindered the self-advocacy of working women was the lack of job security, which sometimes caused them to retreat from their rights and lose self-advocacy. Another identified theme was medial barriers. Ghafari et al. reported that the reasons for MS patients' and their families' dissatisfaction with the rehabilitation staff are as follows: indifference to the patients and their opinions, lack of emotional support and empathy, lack of honesty, not being a good listener, lack of respectful behavior, instilling fear and frustration, poor education/information/correct and timely diagnosis, and negligence in care [17]. Ghanati et al. pointed to the high economic burden of MS and insurance problems with drug costs [18]. This finding is consistent with our results. In our study, most women reported physicians' negligence, lack of empathy, and limitations, and high costs of medications as barriers to self-advocacy based on their experiences.
Another issue was the environmental barriers, including limited access to facilities, transportation problems, and climatic conditions, which restricted women from pursuing their rights and having self-advocacy. It is important to identify and address the barriers that people with MS face during the service delivery, as it helps to improve outcomes continuously [19]. The physical barriers of the learning environment, the lack of trust in teachers, and their inattention to the special conditions of the patients were other environmental barriers that led to anxiety and stress in them and overlooking their voices and needs. Some researchers believe that the institution of education, like the institution of family, is associated with a lot of stress and can cause problems [20]. Although support from the people around can play a very important role in the health and quality of life of MS patients, it can have a negative effect; for example, high empathy can be considered by the patient as pity [21] which can deprive the patient of receiving help in the necessary circumstances and of psychological, emotional, informational and interactive support [22]. This issue was also reported in the present study. Women, in some cases, due to fear of being pitied and judged, not only did not act on their rights and self-advocacy but also preferred to be silent and do nothing.

Another finding of this study was the psychological barriers. Psychological effects of MS include excitement, anxiety, isolation, seclusion, and secrecy [13]. According to Hesseini, psychological consequences of MS arise in response to the disease process, in response to the diagnosis, and due to drug side effects [23]. This classification can also be seen in the present study. Jafari Jozani et al. reported that people with MS tend to be pessimistic and negatively oriented towards life due to psychological disorders [24]. These findings are consistent with our results. Kadkhodai et al. reported the positive relationship of women's selfimportance with their sense of happiness and quality of life. However, self-importance is a new concept for Iranian women, and there is a need for changes in their thought, emotions, and lifestyle patterns to understand and accept it. The women in our study often forgot to pay attention to themselves, and their lifestyle was focused on MS, family, children, and work. In this regard, these findings are consistent with our results.

Considering the effects of family, society, workplace, and learning environment as external factors and psychological, physical, and behavioral issues of the individual as internal factors, the creation of any inhibitory effect by the mentioned factors can lead to limitation of activity and participation of women with MS or affect their performance and physical conditions. Providing educational information about the symptoms and course of MS and how to deal with 
it is particularly important in building trust. Providing accurate information through online social media immediately after diagnosis [26], having the proper social support and appropriate information can play an important role in confronting MS women with the mentioned barriers and realizing their self-advocacy.

\section{Ethical Considerations}

Compliance with ethical guidelines

This study was approved by the Ethics Committee of the Ahvaz Jundishapur University of Medical Science (Code: IR.AJUMS.REC.1398.165). All ethical principles are considered in this article. The participants were informed about the purpose of the research and its implementation stages. They were also assured about the confidentiality of their information. They were free to leave the study whenever they wished, and if desired, the research results would be available to them.

\section{Funding}

This study was MA. thesis of the first author at the Department of Rehabilitation Management, School of Rehabilitation Sciences, Ahvaz Jundishapur University of Medical Sciences, Ahvaz.

Authors' contributions

Conceptualization: Sara Bachari, Roya Ghasemzadeh, Ghazaleh Mandani; Methodology: Ghazaleh Mandani, Roya Ghasemzadeh, Shadab Shah Ali; Research, editing, finalization, review and analysis: All authors; Drafting: Sara Bachari, Ghazaleh Mandani.

\section{Conflict of interest}

The authors declared no conflict of interest.

\section{Acknowledgments}

All women participating in this study, who had shared their time and experiences with us, and all the staff of the Khuzestan MS Association, We are very grateful to Nedamat for their cooperation in conducting this research. 
مقاله بزوهشى

شناسايى موانع خُودمافعدكَى در زنان مبتالا به مالتيّل اسكلروزيس اسثان خوزستان: تحليل محتواى كيفى ميناسي

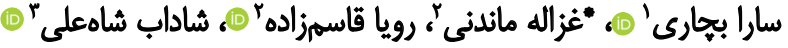

1. كروه مديريت توانبخشى، دانشكده علوم توانبخشى، دانشكاه علوميرشكى جندي تشايور اهواز، اهواز، ايران.

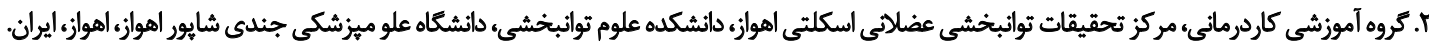

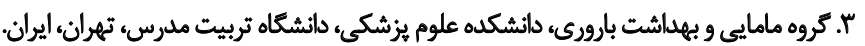

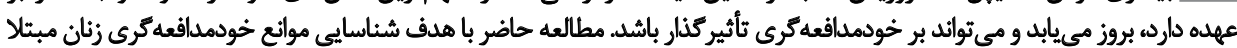

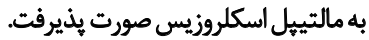

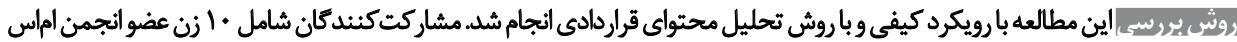

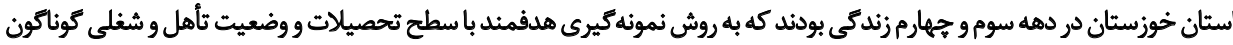

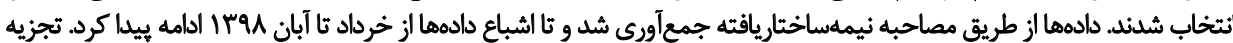

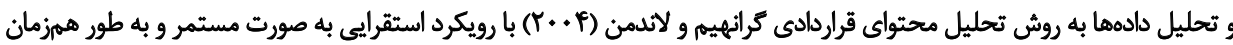

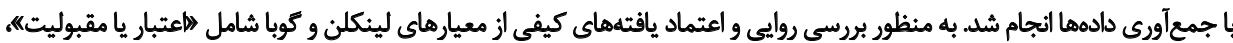

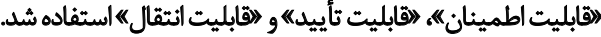

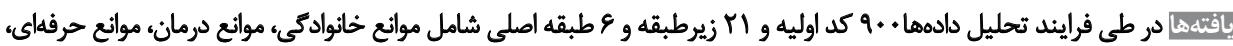

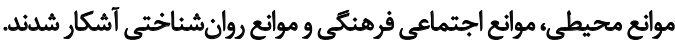

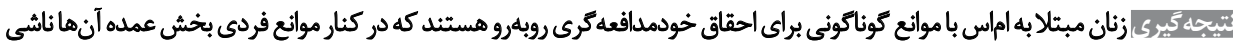

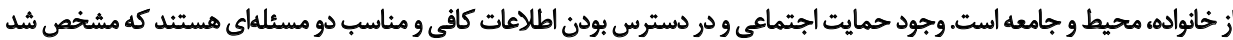

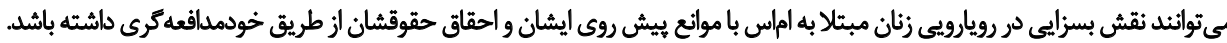

\section{تاريخ دريافت: 10 دى 1499

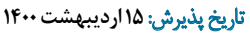

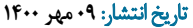

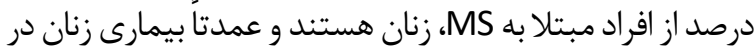

مقدمه

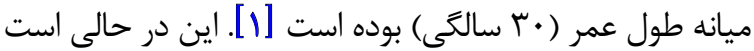
كه زنان در حفظ و ارتقاى كيفيت زندكى داراى نقش مونى مؤثرى

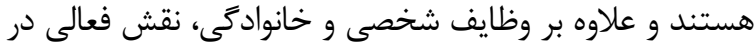

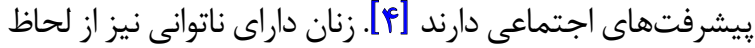

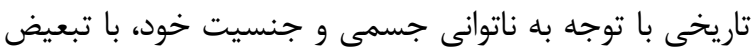

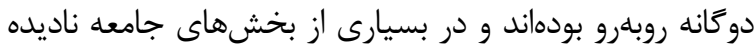

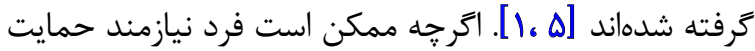

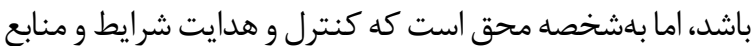

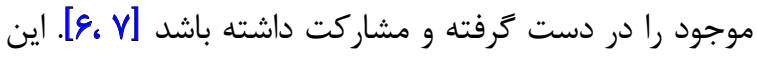
مالتيِّل اسكلروزيس' (MS) بيمارى خود ايمنى، ناتوان كننده و مزمنى است كه با تأثير بر سيستم عصبى مركزى موجب

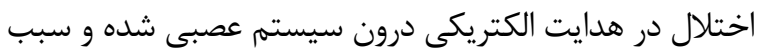

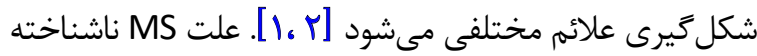

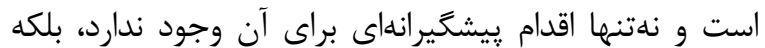

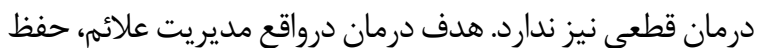

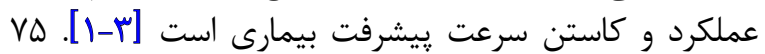

1. Multiple Sclerosis (MS) 
ابتلا به اماس داشتند. انتخاب نمونهها با روش هدفمنده و و با تحري

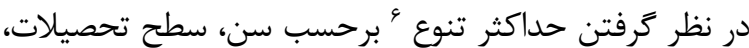

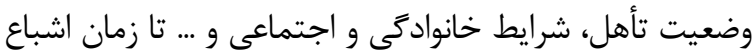

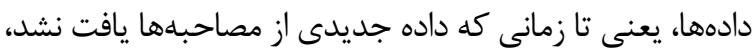

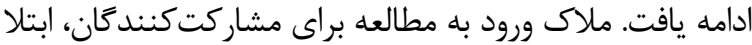

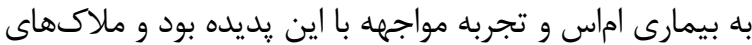

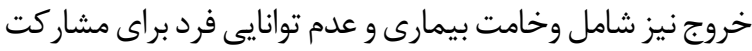

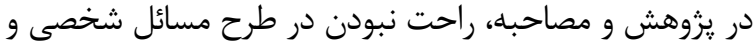

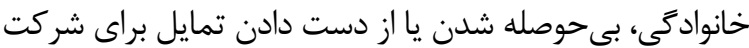

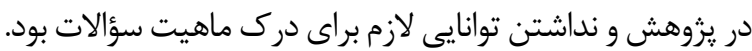

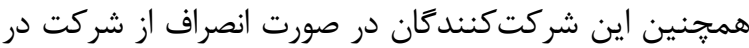
مصاحبه، از مطالعه خارج مىشد شيند.

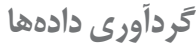

دادهها در اين تحقيق با استفاده از مصاحبه عميق و

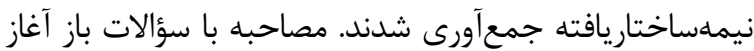

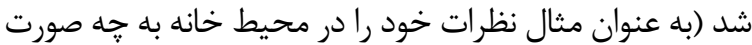

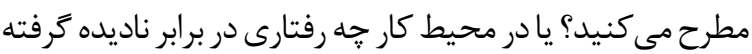

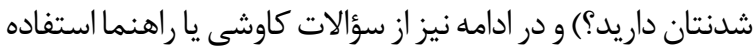

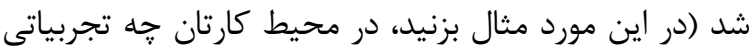

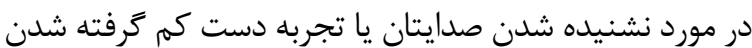

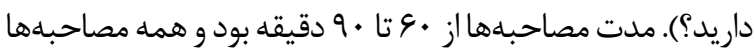

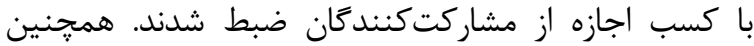

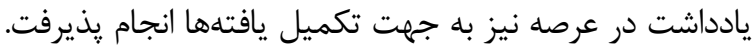

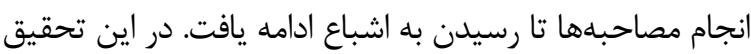

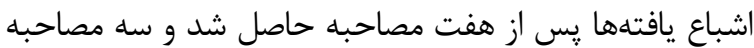

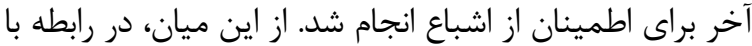

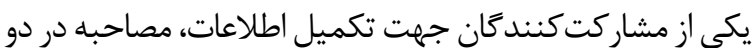

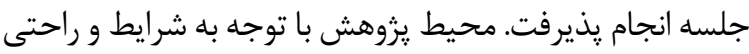

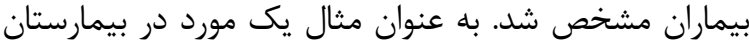

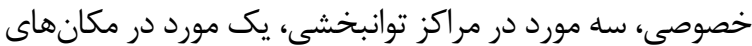

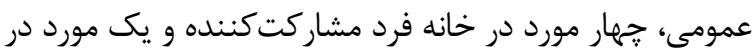

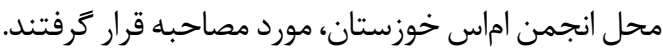

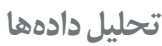

جهت تجزيه و تحليل دادهها از روش "اتحليل محتواى كيفى بر

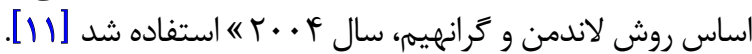

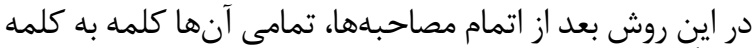

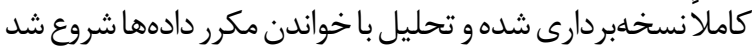

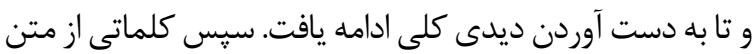

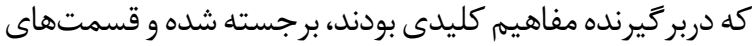

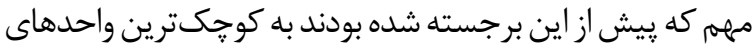

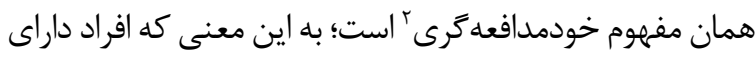

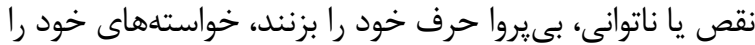

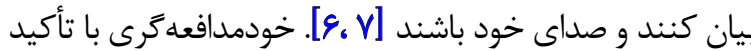

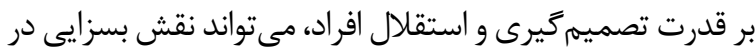

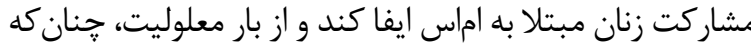

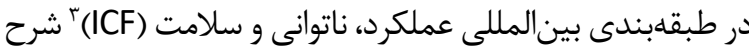

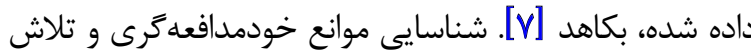

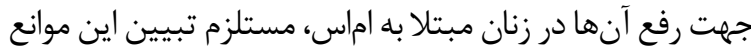

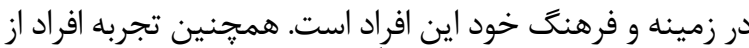

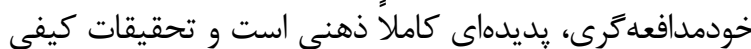

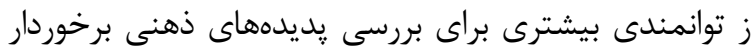

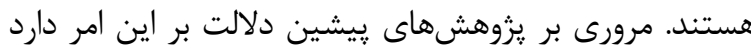

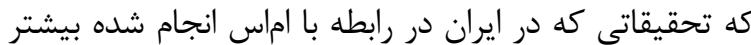

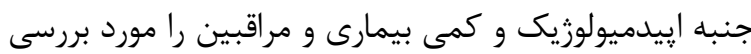

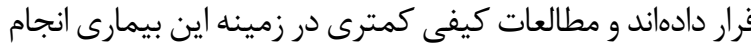

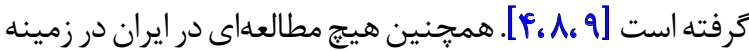

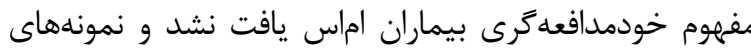

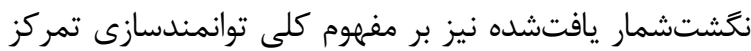

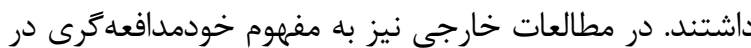

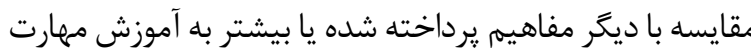

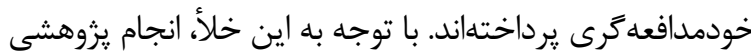

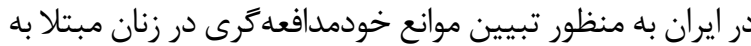

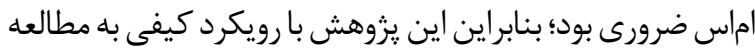

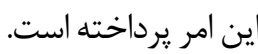

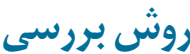
طرح مطالعه

مطالعه حاضر، مطالعهاى كيفى از نوع تحليل محتواى عرفى إنى

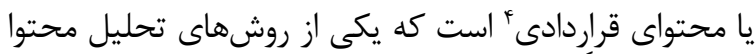

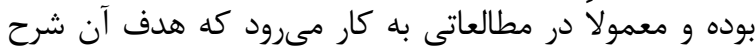

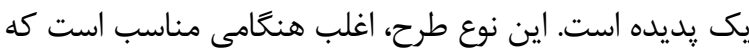

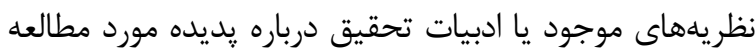

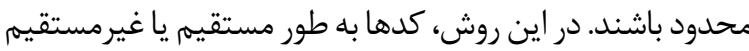

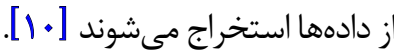

$$
\text { مشار كت كنند }
$$

جامعه يزوهش در اين مطالعه اعضاى انجمن اماس استان

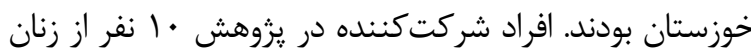

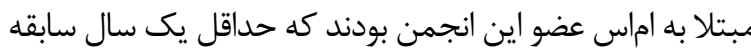

\section{Self advocasy}

3. International Classification of Functioning, Disability and Health (ICF)

4. Conventional content analysis 
است. براى برخى از زنان نيز عدم يذيرش اماس از سوى خانواده

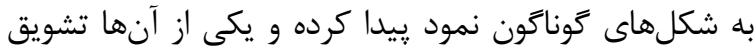

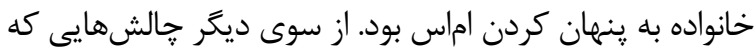

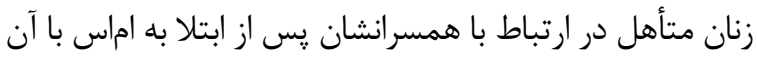

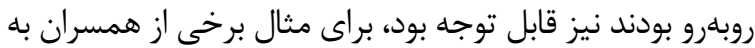

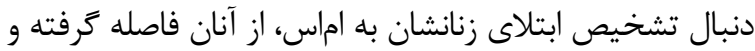

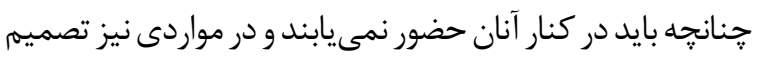

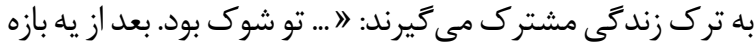

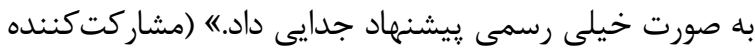

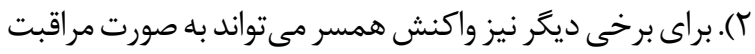

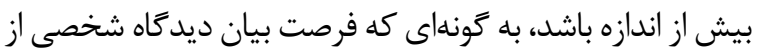

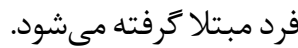

موانع حرفهاى: زيرطبقات النداشتن امنيت شغلى"، "امؤثر

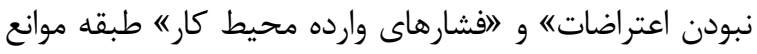

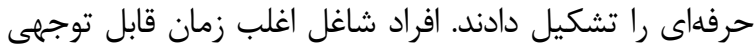

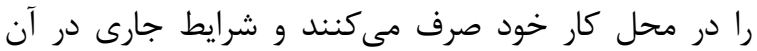

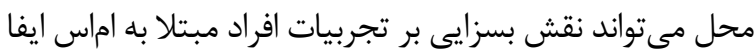

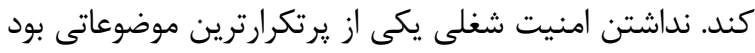

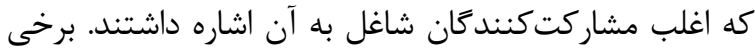

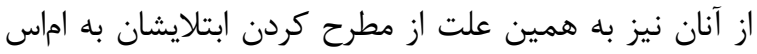

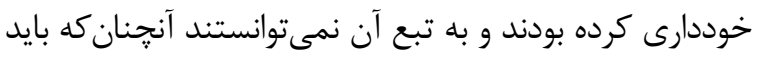

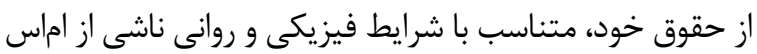

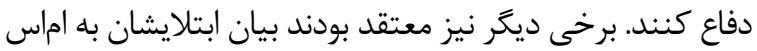

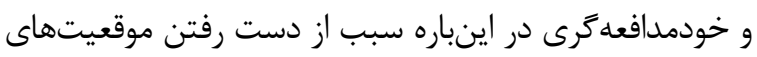

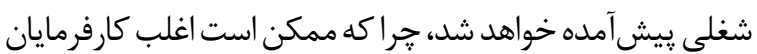

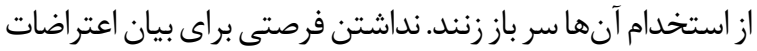

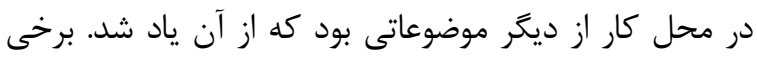

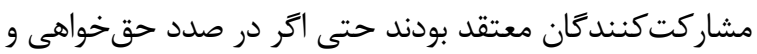

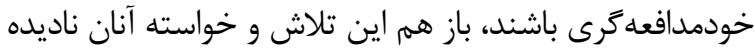

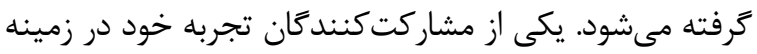

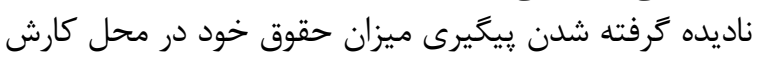

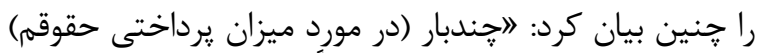

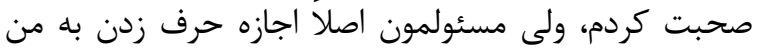

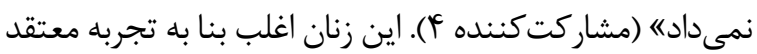

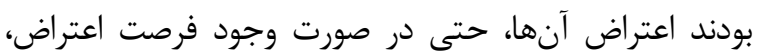
شنيده نخواهد شد.

موانع درمان: "كوتاهى يزشكى"، العملكرد ضعيف كادر

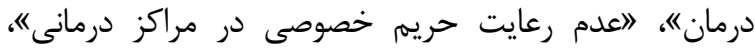

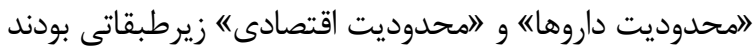
كه طبقه موانع درمان را تشكيل دادند.

نحوه كرفتن تشخيص و بيان آن از سوى يزشك مى تواند سبب

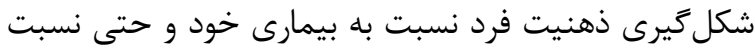

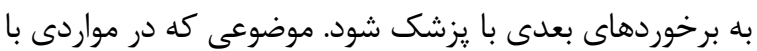

تشكيل دهنده و معنى دار (كد) شكسته شدند. جدولى سهستونه

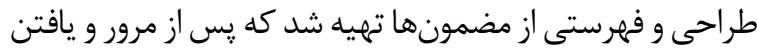

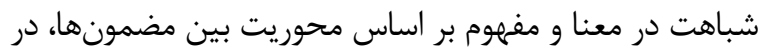

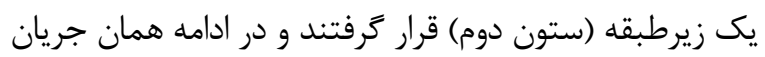

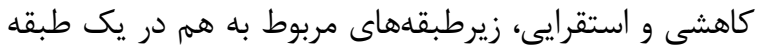

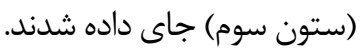

صحت و استحكام دادهها

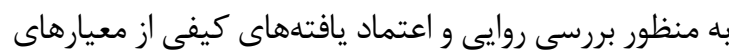

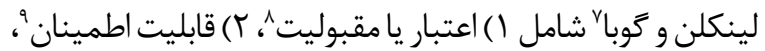

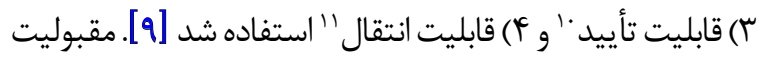

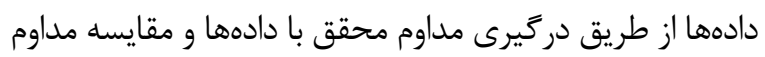

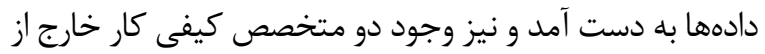

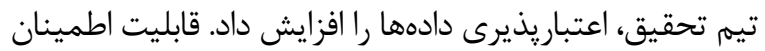

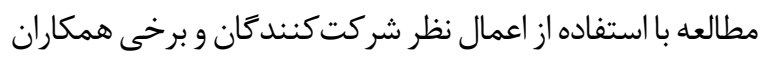

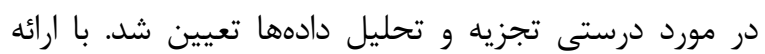

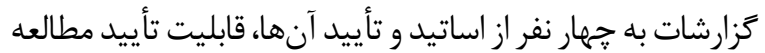

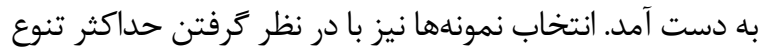

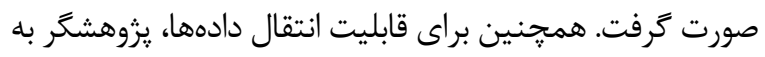

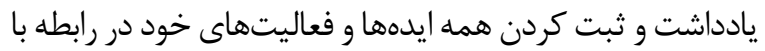

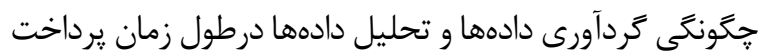

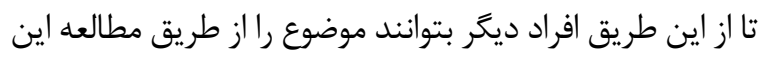

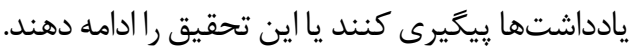

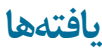

مشاركت كنندكان، زنانى شاغل يا خانهدار، در دها سوم سوم يا

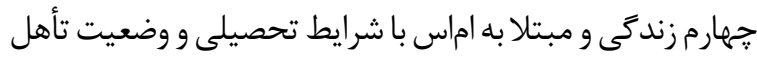

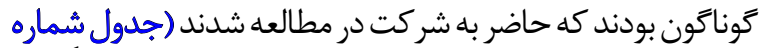

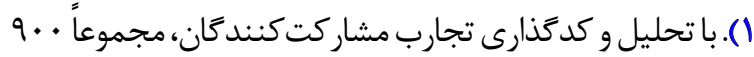

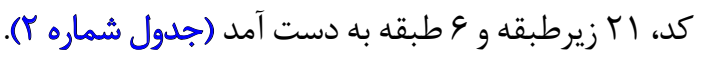

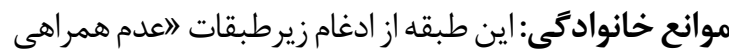

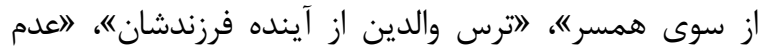

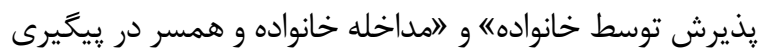

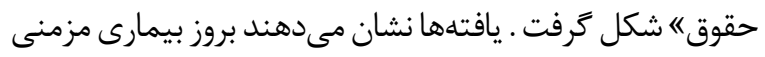

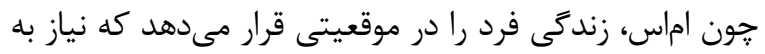

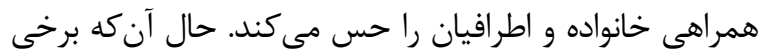

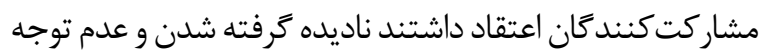

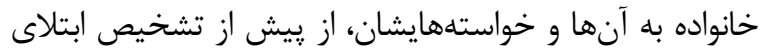

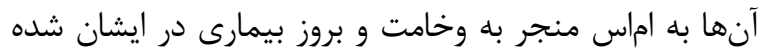

11. Transferability 
جدول ا. مشخصات جمعيتشناختى مشاركت كندكان

\begin{tabular}{|c|c|c|c|c|c|c|}
\hline مدت ابتلا به اماس (سال) & شهر & وضعيت تأهل & شغل & تحصيلات & سن & شماره \\
\hline if & خرمشهر & متاهمل & شاغل & كارشناسى & is & 1 \\
\hline r & اهواز از & متأهل & محصل & دائشجو & rq & r \\
\hline r & أبادان & | مجرد & شاغل ل & دييله & ro & $r$ \\
\hline 8 & بهيهان & متأهل & شاغل ل & كارشناسى & Pl & r \\
\hline 1 & أبادان & متأهل & خانهالر & سيكل س & rA & $\Delta$ \\
\hline r & خرمشهر & متأهل & خانهالر & راهنمايى & r. & 8 \\
\hline$\Delta$ & آبادان & متأهل & 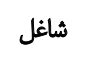 & كارشناسى & r. & $r$ \\
\hline v & آبادان & متاهل & خانهلار & كارشناسى & $\pi$ & $\wedge$ \\
\hline 1 & خرمشهر & متأهل & خانهالر & راهنمايى & rA & 9 \\
\hline 9 & ايذه & مجرد & شاغل & كارشناسى & $r$ & 1. \\
\hline
\end{tabular}

بلدويزه آنهايى كه دور از مركز استان سكونت دارند، با آن روبهرو

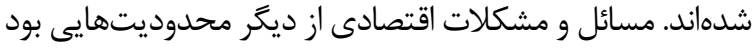

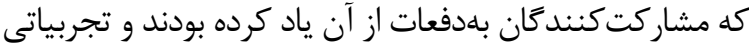

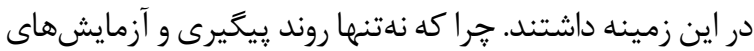

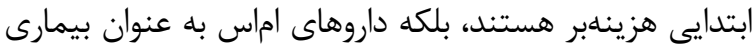

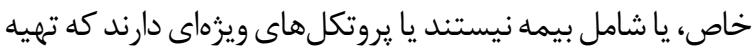

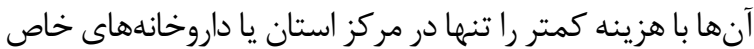

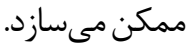

موانع محيطى: طبقه موانع محيطى از ادغام زيرطبقات

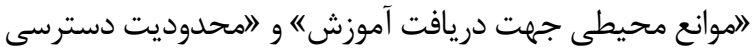

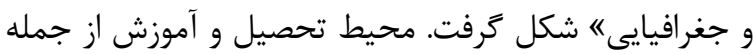

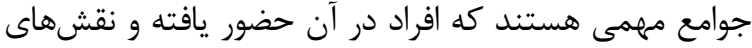

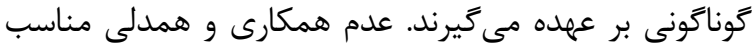

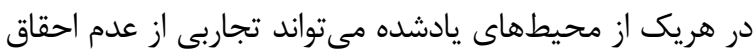

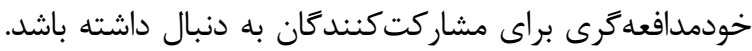
يكى از مشاركت كنند

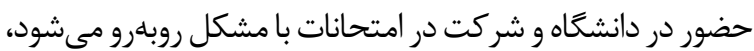

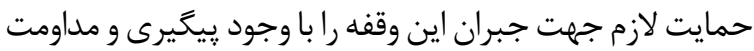

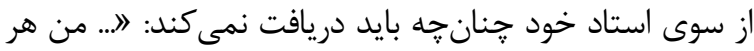

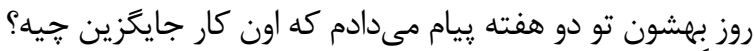

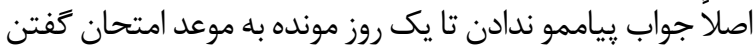

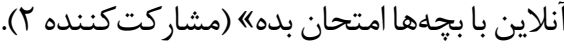

تعدادى از مشاركت كنندكان نيز از محدوديت و محروميت

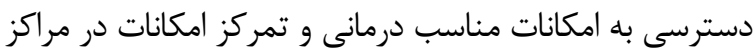

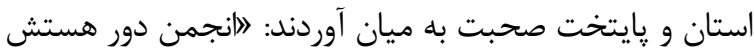

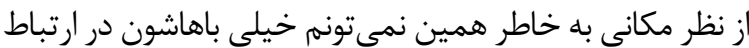

برخورد نامناسب يزشك در هنحام بيان تشخيص، سبب شوكه

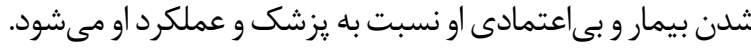

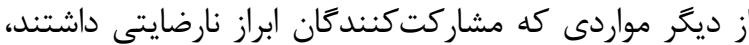

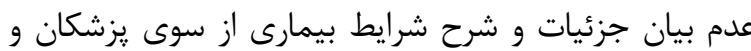

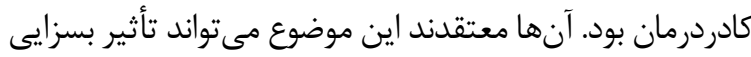

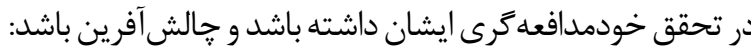

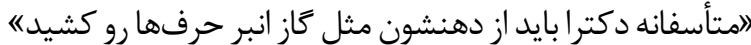

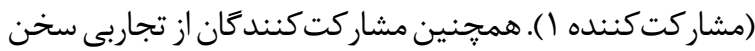

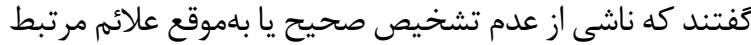

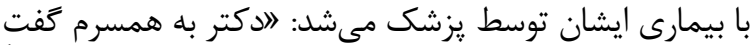

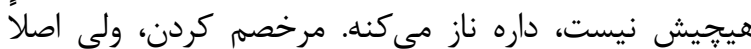

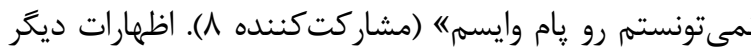

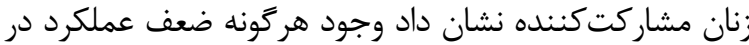

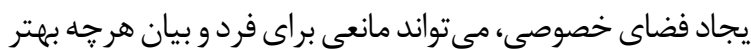

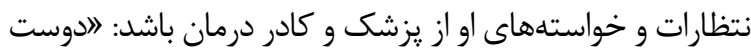

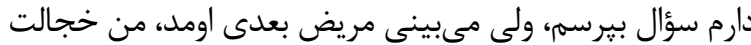

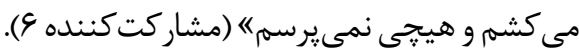

محدوديت داروها يكى ديخر از زيرطبقات مطرحشده بود.

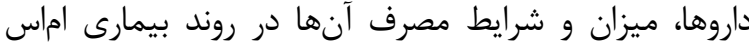

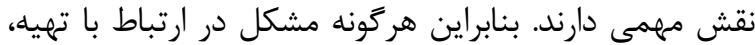

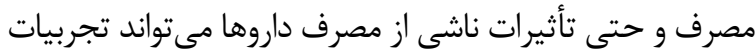

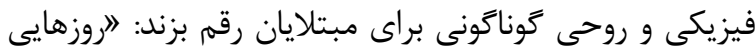

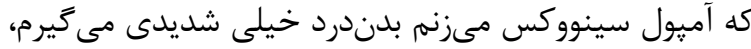

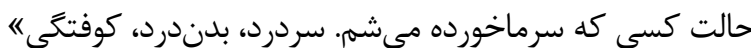

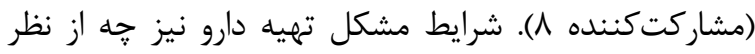

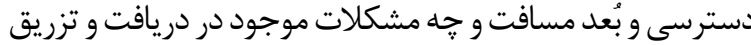

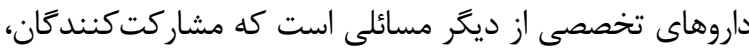


جدول r. طبقات و زيرطبقات

\begin{tabular}{|c|c|}
\hline طبقات & زيرطبقات \\
\hline موائع خانوادكىى & 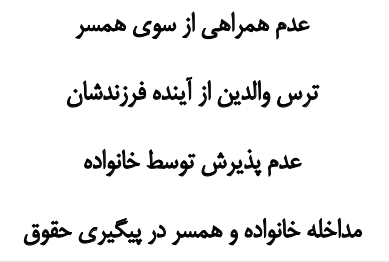 \\
\hline موانع حرقهاى & 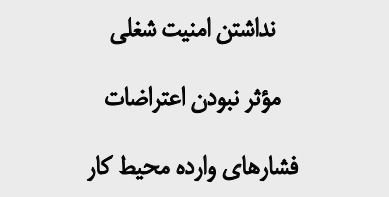 \\
\hline موانع درمان & 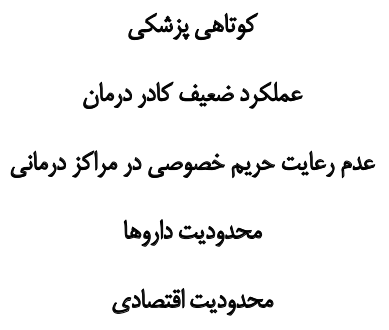 \\
\hline موانع محيطى & $\begin{array}{l}\text { موائع محيطى جهت دريافت أموزش } 2 \text { محلوديت دسترسى و جنرافياييى }\end{array}$ \\
\hline موانع اجتماعى فرهنكى & انكى و قضاوت \\
\hline موائع روانشناختى & 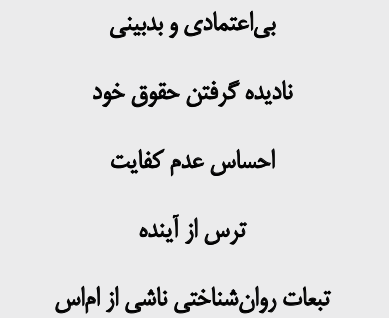 \\
\hline
\end{tabular}

منفى موجود در جامعه، قضاوتها منجر به برانخيختن ترحم در

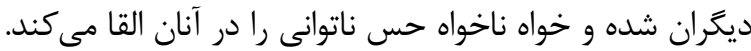

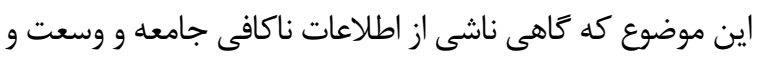

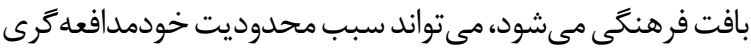

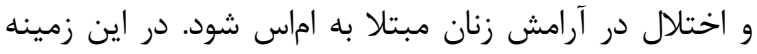

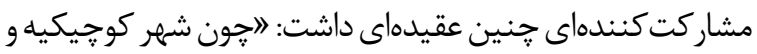
تو زبونا مى جرخه. اخر شهر بزرتى آدم گمم مىشه توى شهر و ناشناس هسته (مشار كت كننده () ).

موانع روانشناختى: زيرطبقات لابىاعتمادى و بدبينى به

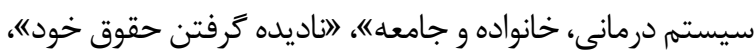

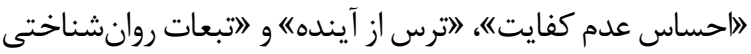

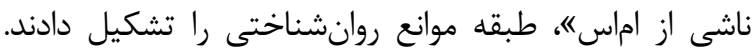

باشه..." (مشاركت كننده ()؛ لهيج كدوم از شهرهاى ايران امكانات

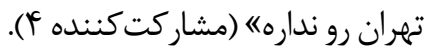

موانع اجتماعى فرهنگى: زيرطبقات 》انت و قضاوته و و

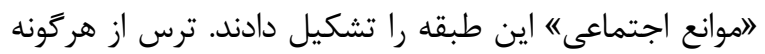

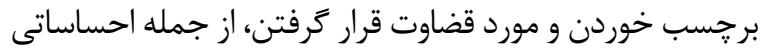

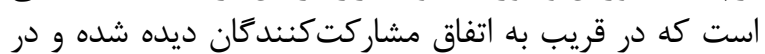

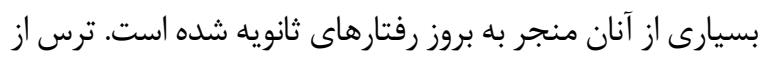

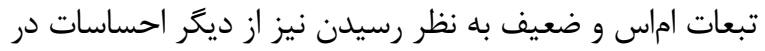

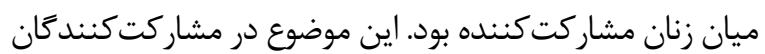

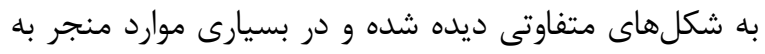

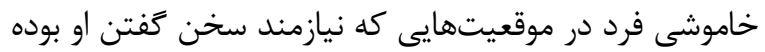
است، مىشد. مشاركت كنند 
مشاركتكنندهاى دليل اين مسئله را انخشتنما شدن و احتمالٍ

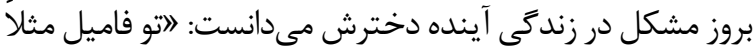

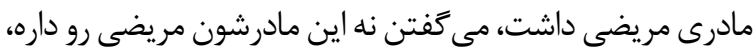

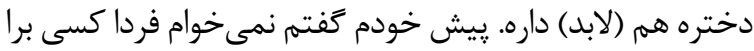

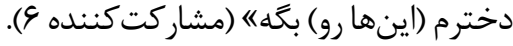

ترس از آينده مبهمه نيز يكى از موارد يرتكرار در ميان

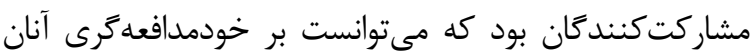

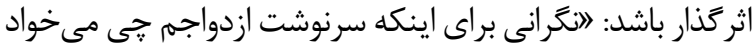

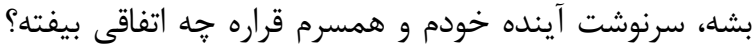

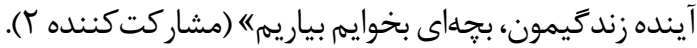

مطالعه حاضر با بررسى و كشف موانع موجود در مسير

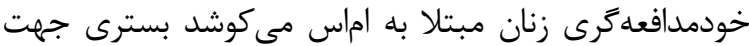

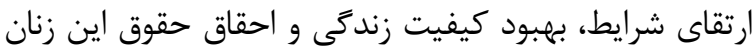

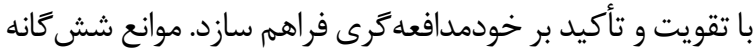

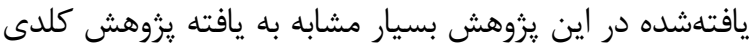

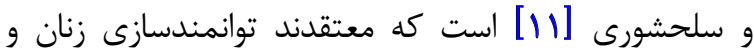

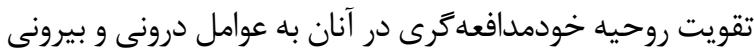

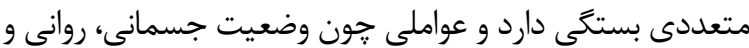

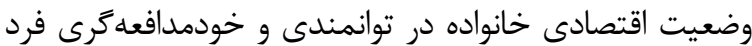

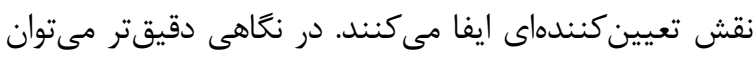

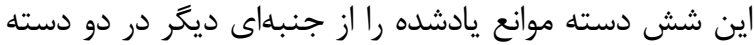

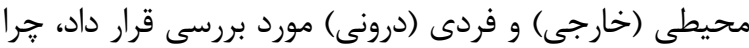

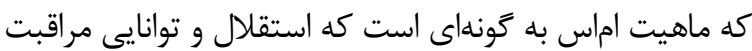

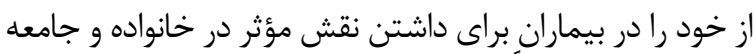

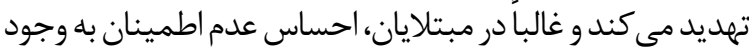

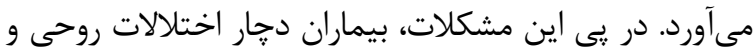

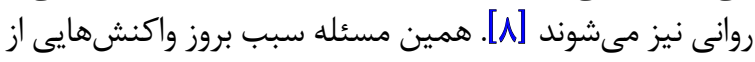

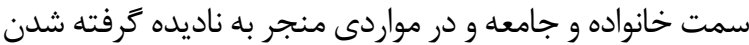

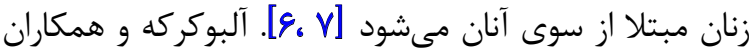

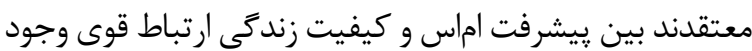

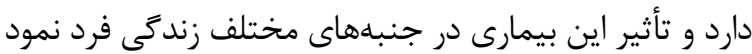

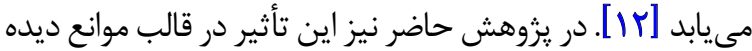

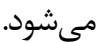

موانع خانواد كى به عنوان يكى از موانع بهدست آمده بيان مي دارد

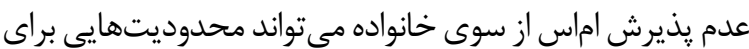

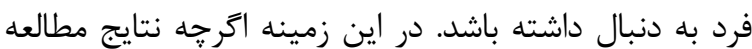

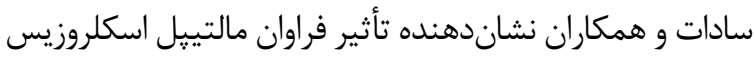

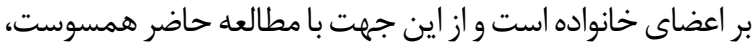

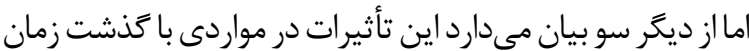

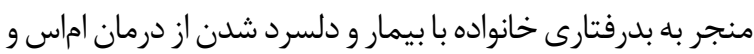

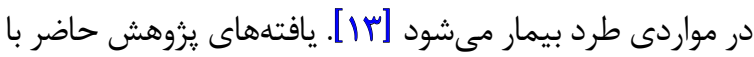

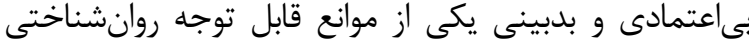

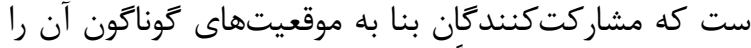

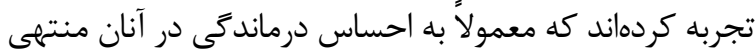

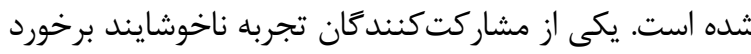

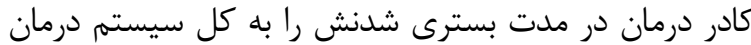

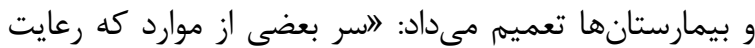

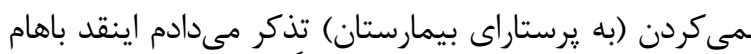

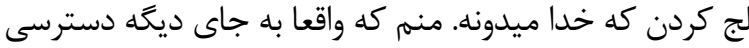

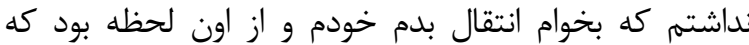

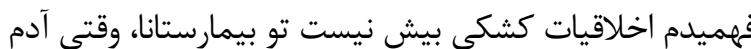

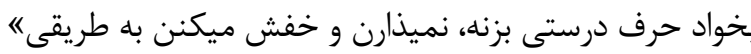

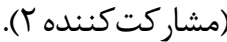

وجود تجربه مخالفتهاى قبلى خانواده نسبت بهبيان خواستهها

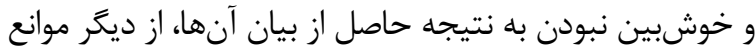

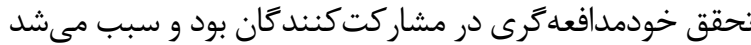
اين زنان زيخيرى و اصرارى بر خواستههايشان نداشته باشندان

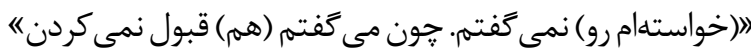

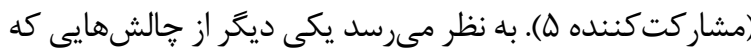
كريبان گير مشاركت كنند

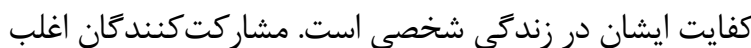

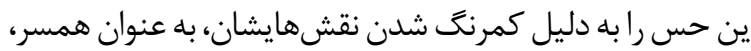

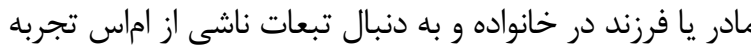

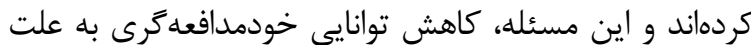
حس عدم كفايت را براى آنان به دنبال داشته است خت

اغلب اين تجربيات و خالشها منجر به بعرغبت شدن نس نسبت

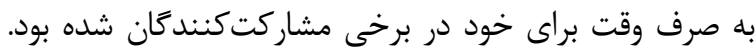

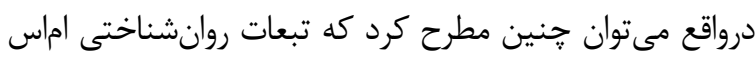

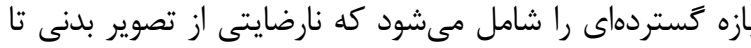

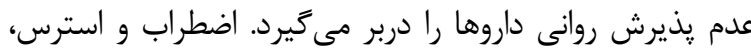

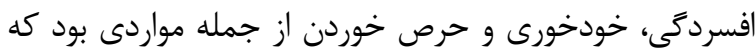

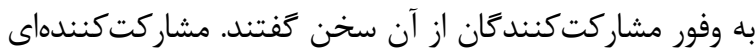

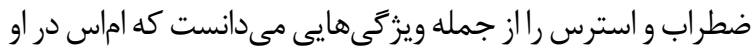

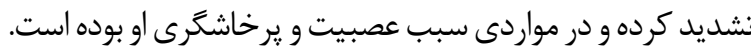

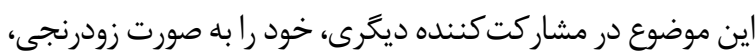
درونريزى احساسات و حساسيت رفتارى نشان داده است.

جالشهاى ناشى از اين موارد و ابهام در روند نامشخص اماس،

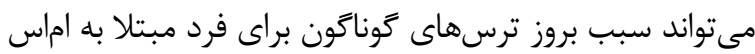

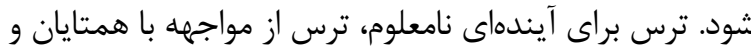

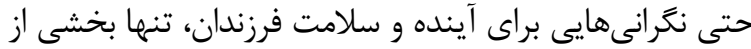

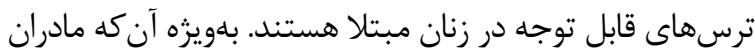

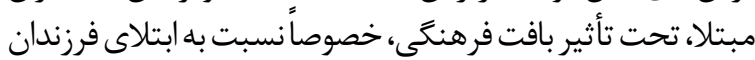

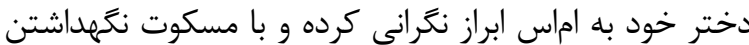

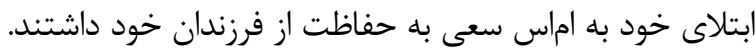


دادن به بيمار و فقدان همدلى و توجه كافى نار اضى بودند و همين

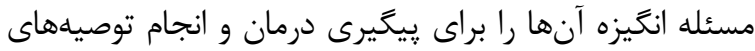

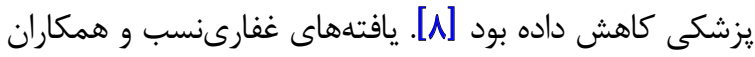

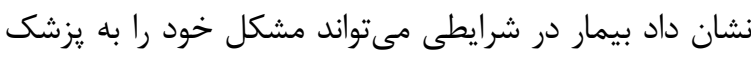

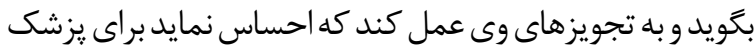

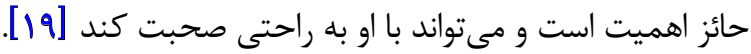

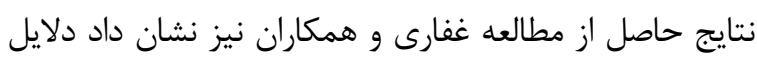

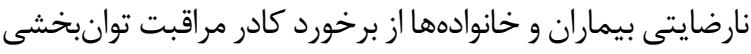

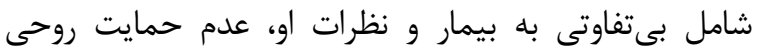

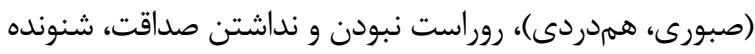

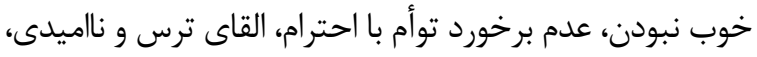

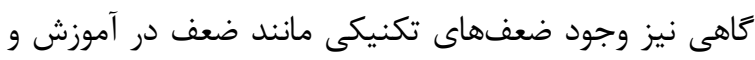

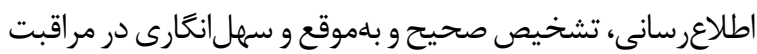

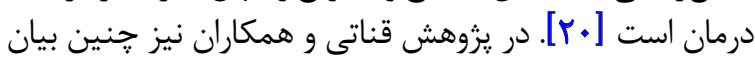

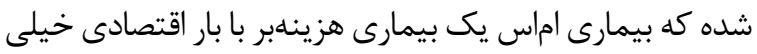

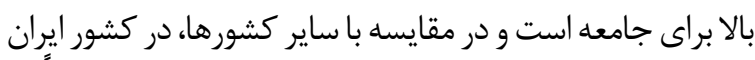

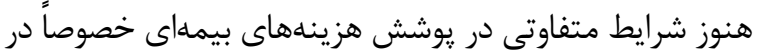

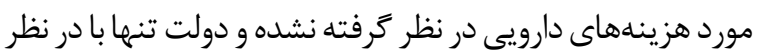

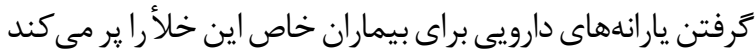

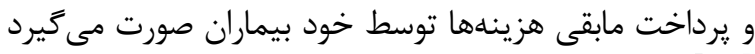

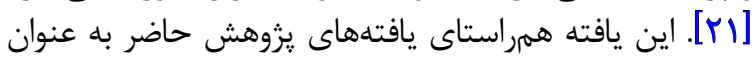

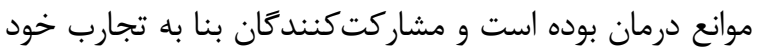

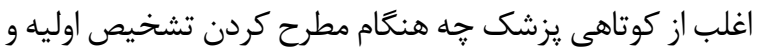

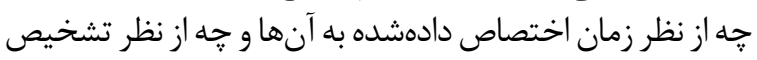

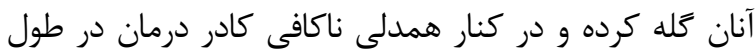

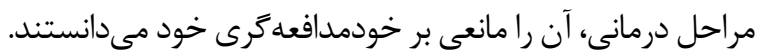

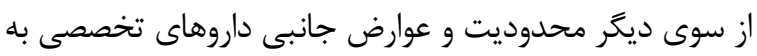

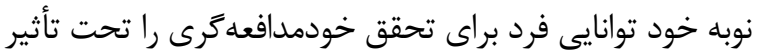

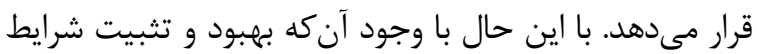

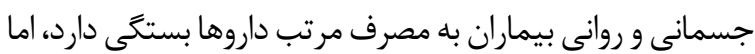

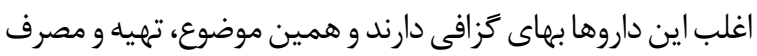

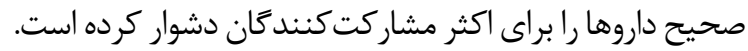

يكى از موضوعاتى كه در حيطه موانع محيطى جاى ميى گيرد،

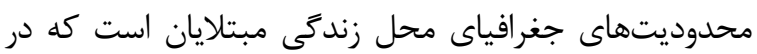

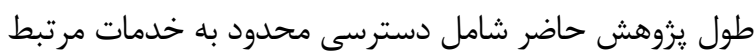

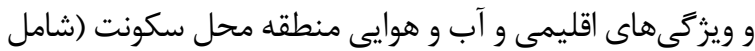

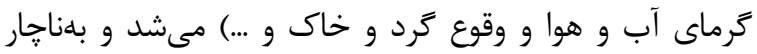

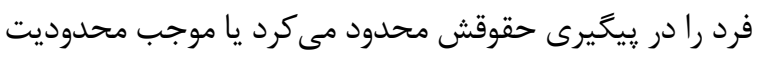

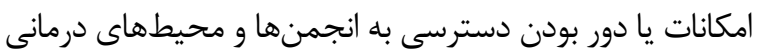

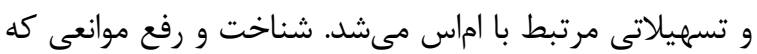

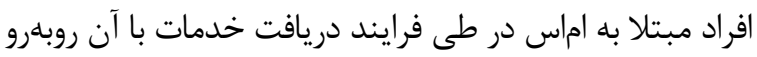

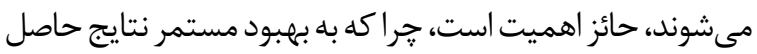

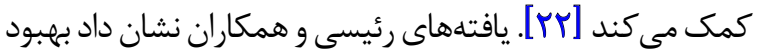
زيرساختهاى شهرى، كاهش تراكم ساختمانى، كنترل مسائل
اين موارد مغايرت دارد و اغلب مشاركت كنندكان با كذشت زمان،

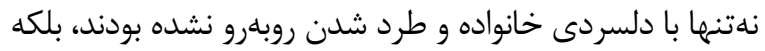

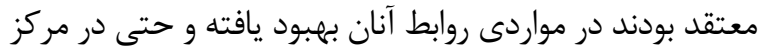

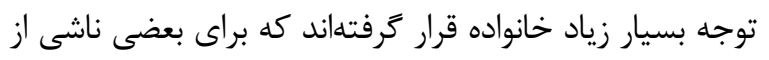

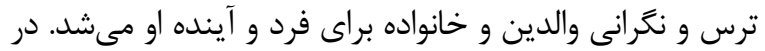

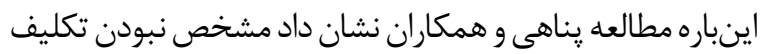

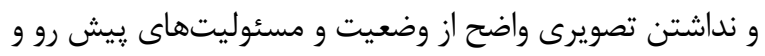

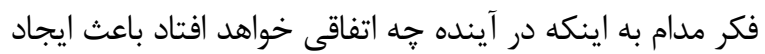

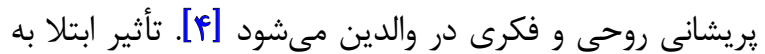

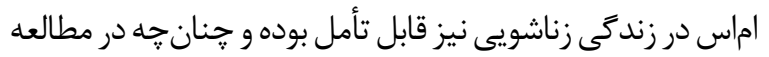

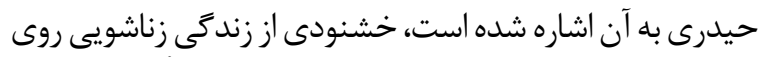

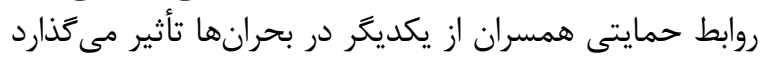

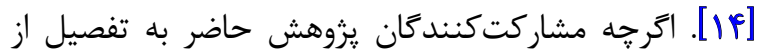

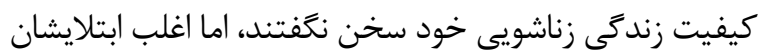

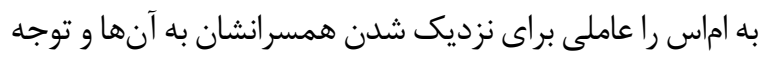

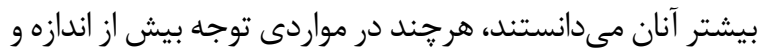

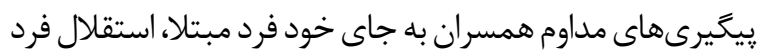
براى دفاع از خود را تحت تأثير قرار داده بود.

موانع حرفهاى از ديكر يافتههاى اين يزوهش بود. يزوهش

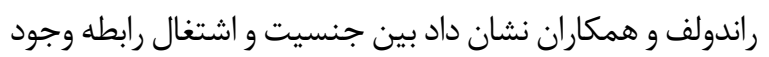

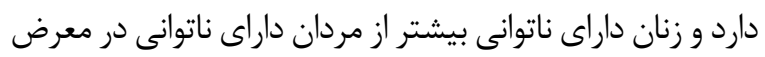

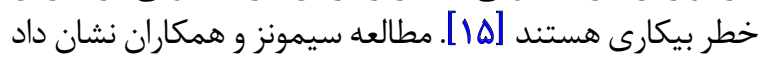

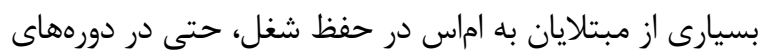

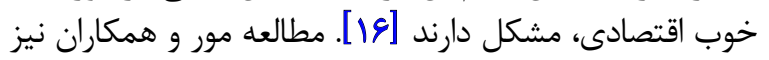

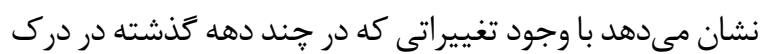

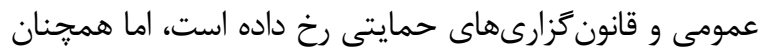

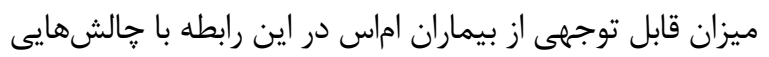

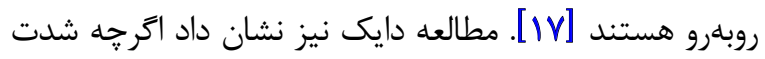

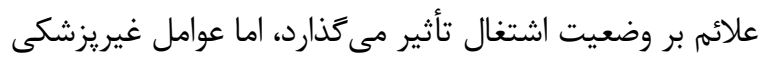

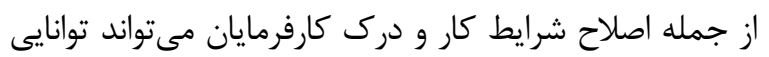

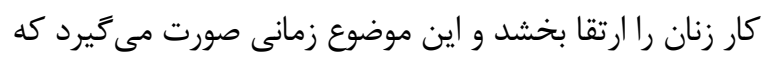

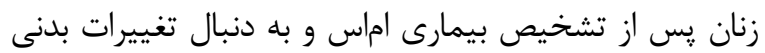

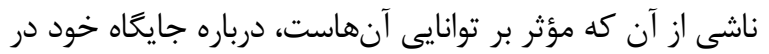

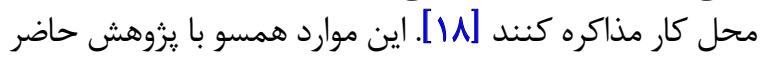

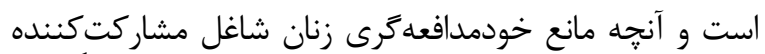

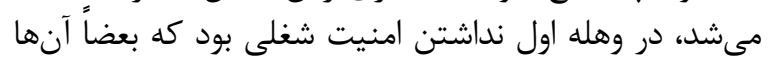

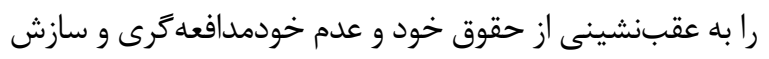

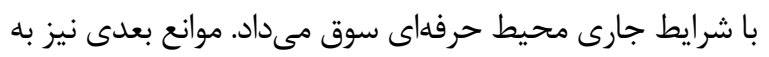

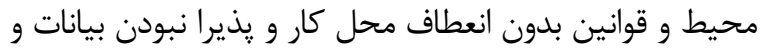
خواستههاى اين زنان مربوط بود.

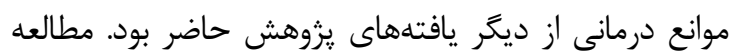

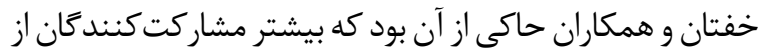

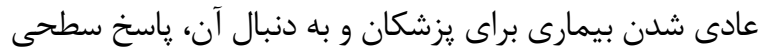




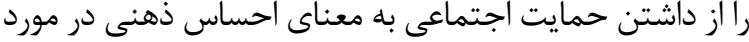

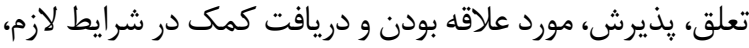

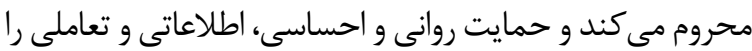

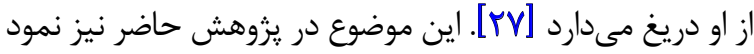

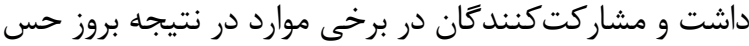

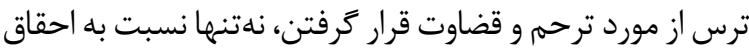

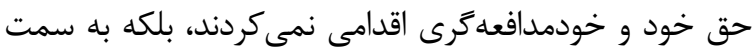

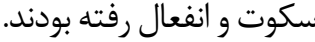

موانع روانشناختى موجود بر سر راه زنان مبتلا به اماس از

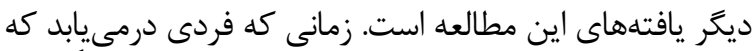

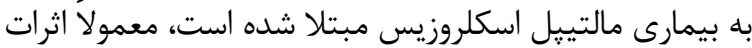

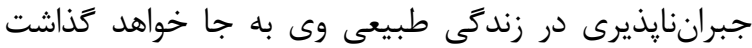

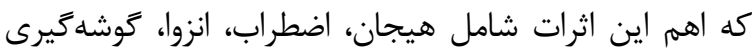

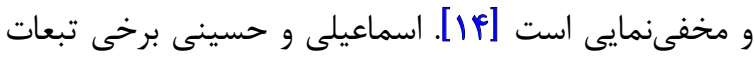

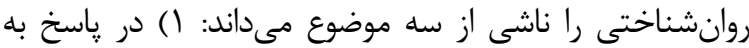

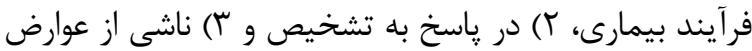

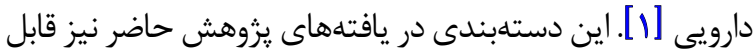

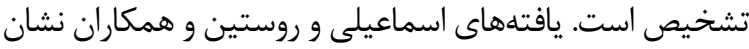

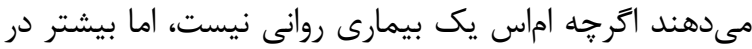

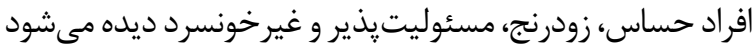

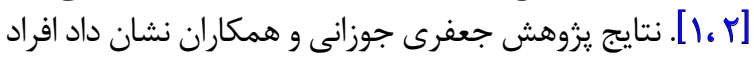

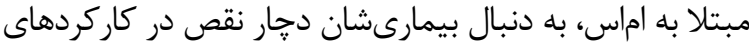

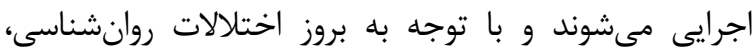

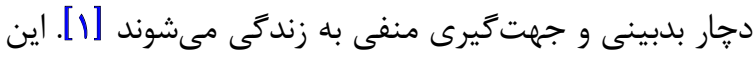

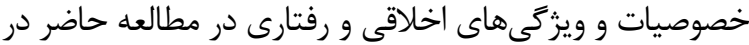

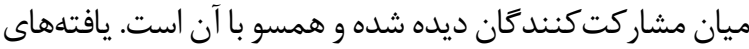

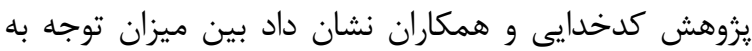

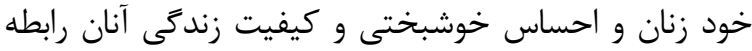

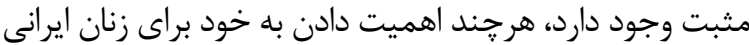

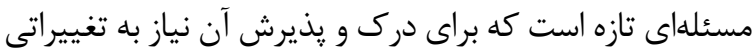

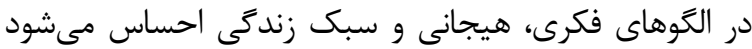

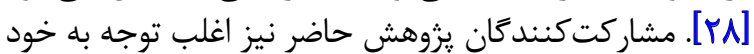

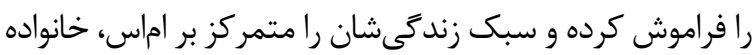

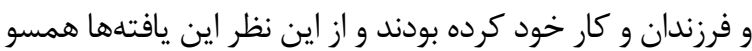
با مطالعه ذكرشده است. تهيه موارد اطلاعرسانى و و آموزشى درد إنى

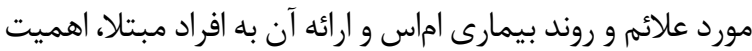

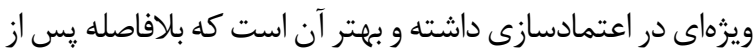

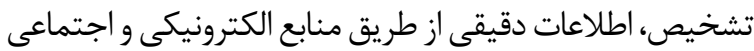

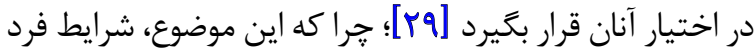

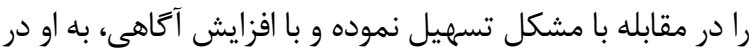
غلبه بر ترسهاى موجود كمك مى كندي
ترافيكى و ارتقاى كيفيت زندگى شهرى مى تواند منجر به بهبود

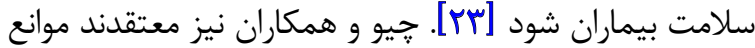

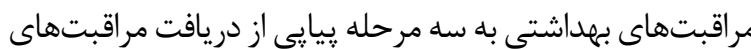

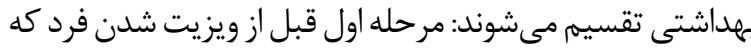

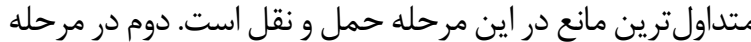

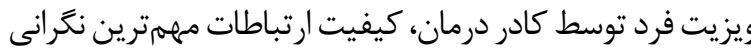

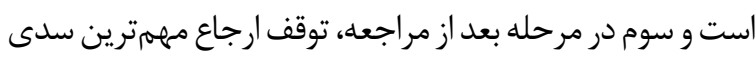

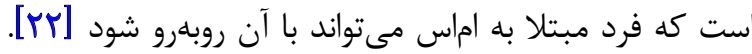

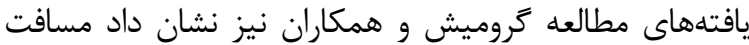

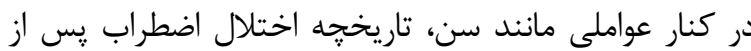

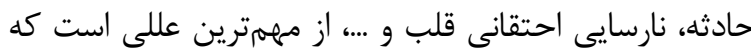

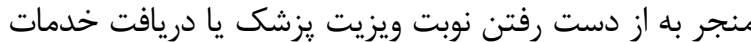

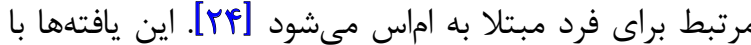

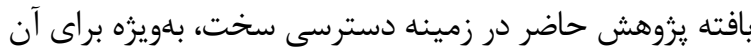

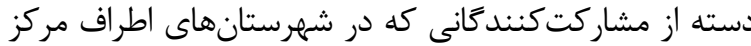

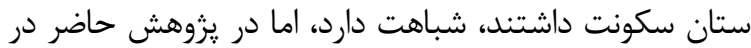

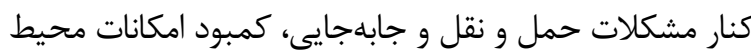

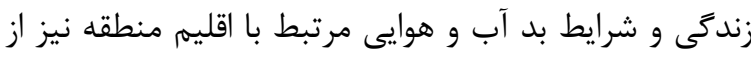

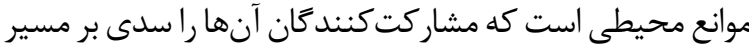
خودمدافعه كرى خود مي دانستند.

محيط آموزشى از ديخر محيطهاى مههم و قابل توجه است كه

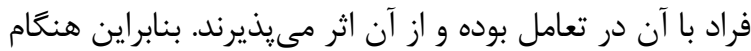

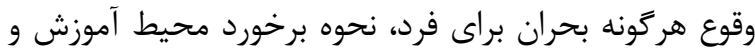

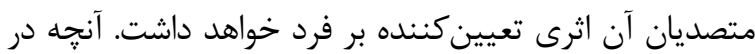

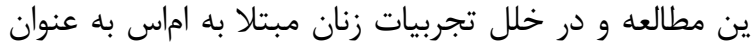

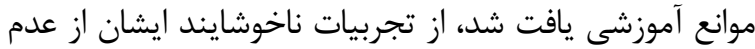

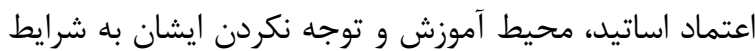

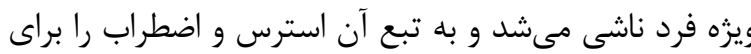

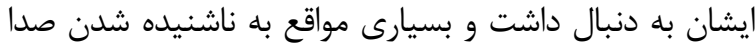

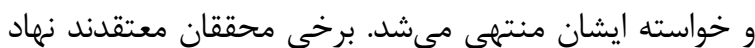

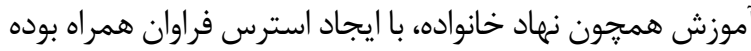

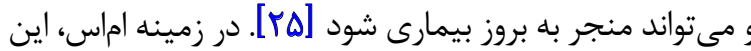

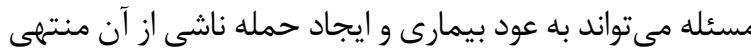

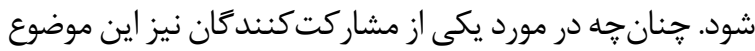

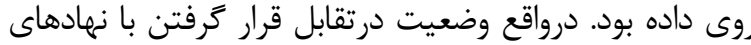

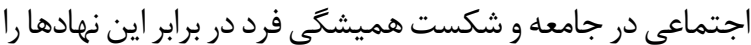

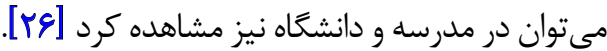
برخى مطالعات نشان مى دهند اكرجه حمايت از سوى اطرافيان

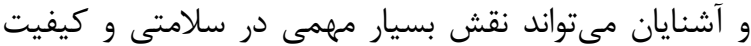

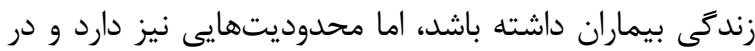

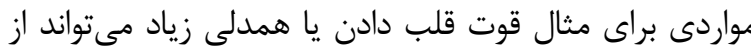

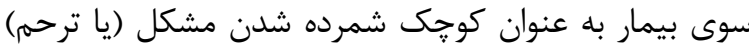

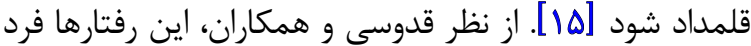




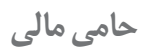

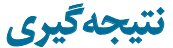

اين مقاله بخشى از باياننامه مقطع كارشناسى ارشد نويسنده

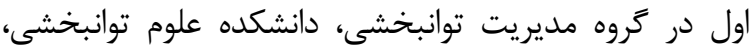

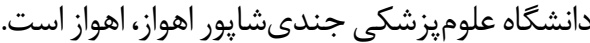

$$
\text { مشار كت نويسند }
$$

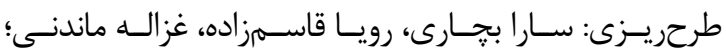

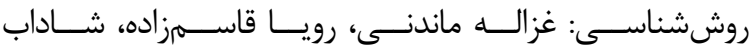

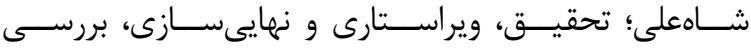

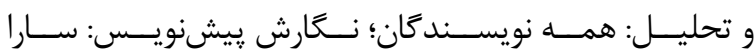

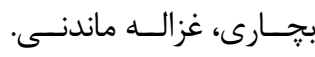

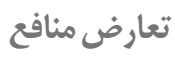

بنابر اظهار نويسندكان اين مقاله تعارض منافع ندارد.

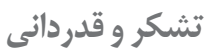

از تمامى زنان مشاركتكننده در اين يزوهش كه بدرن بدون

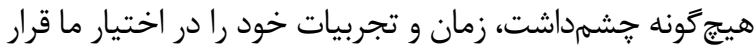

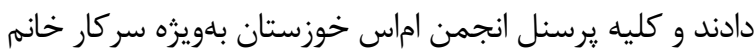

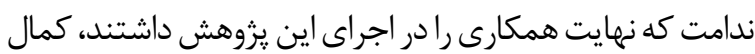

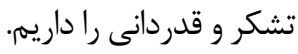

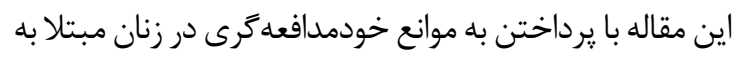

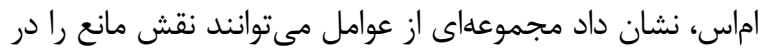

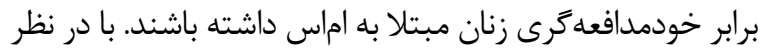

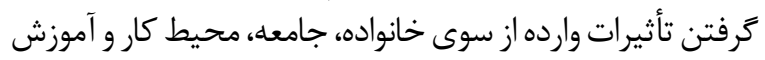

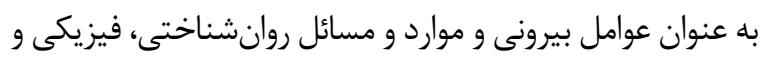

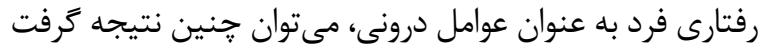

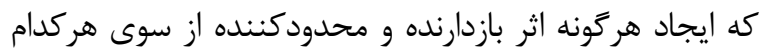

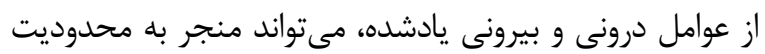

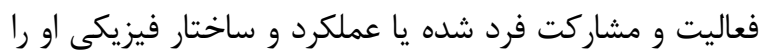

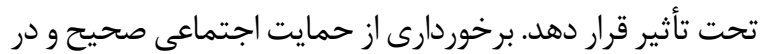

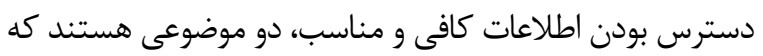

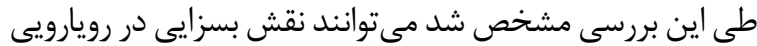

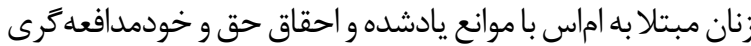

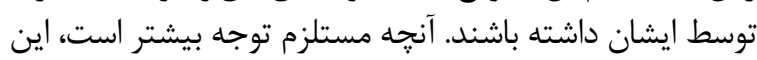

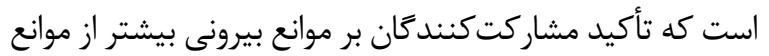

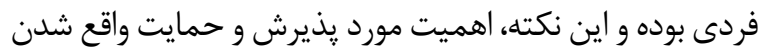

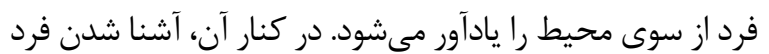

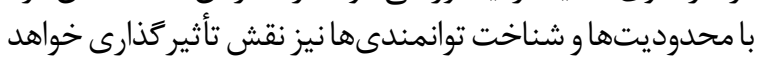

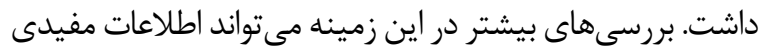
در اين زمينه در اختيار متوليان اين امر قرار دهد.

Letuggow

از محدوديتهاى اين مطالعه مي توان به جند مورد اشاره كرد؛ از

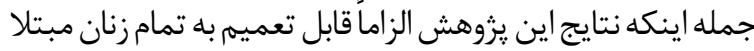

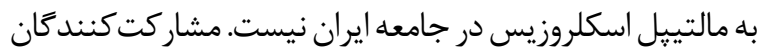

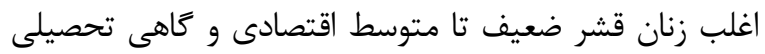

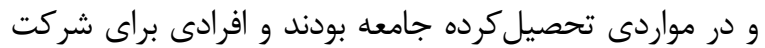

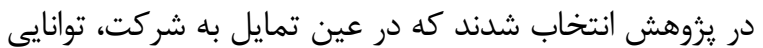

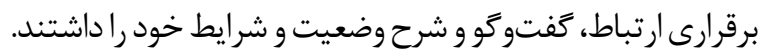

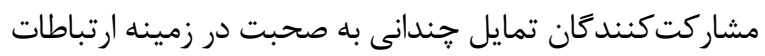

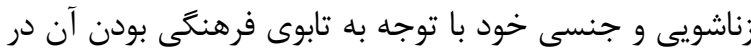

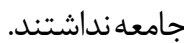

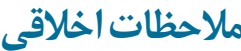

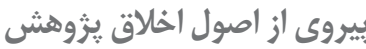

اصول اخلاق در يزوهش مورد تاييد كميته اخلاق دانشخاه

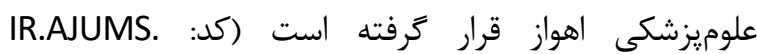

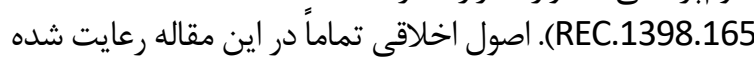
است. شركت كنند

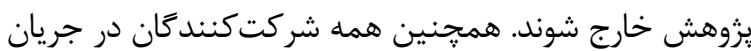

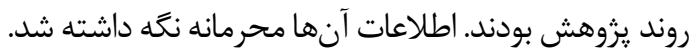




\section{References}

[1] Esmaeili MA, Hosseini FA. Multiple Sclerosis and stressful thought habit. Iranian Journal of Nursing Research (IJNR). 2008; 3(11):25-32. https://vlibrary.emro.who.int/imemr/multiple-sclerosis-and-stressful-thought-habit-2/

[2] Rotstein Z, Hazan R, Barak Y, Achiron A. Perspectives in multiple sclerosis health care: Special focus on the costs of multiple sclerosis. Autoimmunity Reviews. 2006; 5(8):511-6. https://www. sciencedirect.com/science/article/abs/pii/S156899720600019X

[3] Zhornitsky S, Yong VW, Weiss S, Metz LM. Prolactin in Multiple Sclerosis. Multiple Sclerosis. 2013; 19(1):15-23. [DOI:10.1177/1352458512458555] [PMID]

[4] Panahi M, Tazakori Z, Karimollahi M. [Challenges of parents of daughters with Multiple Sclerosis: A phenomenological study (Persian)]. Journal of Health and Care. 2018; 20(2):165-76. [DOI:10.29252/jhc.20.2.165]

[5] Shakouri A, Rafat Jah M, Jafari M. [An analysis of factors affecting women's empowerment components (Persian)]. Women in Development \& Politics. 2007; 5(1):1-26. https://www.sid.ir/en/ Journal/ViewPaper.aspx?ID=101863

[6] Khasnabis C, Motsch KH, Achu K, Al Jubah K, Brodtkorb S, Chervin P, et al. Community-based rehabilitation: CBR guidelines. Geneva: World Health Organization; 2010. https://pubmed. ncbi.nlm.nih.gov/26290927/

[7] Kheftan P, Gholami Jam F, Arshi M, Vameghi R, Khankeh HR, Fathi M. [Explaining the individual problems of women affected by Multiple sclerosis: a qualitative study (Persian)]. Journal of Jiroft University of Medical Sciences. 2017; 3(1):47-54. http:// journal.jmu.ac.ir/article-1-124-en.html

[8] Khftan P, Vameghi R, Khankeh HR, Fathi M, Arshi M, Gholami Jam F. [Exploring therapeutic problems in women with Multiple Sclerosis: A qualitative study (Persian)]. Journal of Qualitative Research in Health Sciences. 2017; 6(1):13-21. http://jqr1.kmu. ac.ir/article_90879.html

[9] Hsieh HF, Shannon SE. Three approaches to qualitative content analysis. Qualitative Health Research. 2005; 15(9):1277-88. [DOI:10.1177/1049732305276687] [PMID]

[10] Graneheim UH, Lundman B. Qualitative content analysis in nursing research: Concepts, procedures and measures to achieve trustworthiness. Nurse Education Today. 2004; 24(2):105-12. [DOI:10.1016/j.nedt.2003.10.001] [PMID]

[11] Kaldi A, Salahshouri P. [The investigation of social support's effect on women empowerment (Persian)]. Journal of Iranian Social Development Studies. 2012; 4(4):7-22. https://jisds.srbiau. ac.ir/article_1871.html?lang=en

[12] Albuquerque C, Geraldo A, Martins R, Ribeiro O. Quality of life of people with Multiple Sclerosis: Clinical and psychosocial determinants. Procedia-Social and Behavioral Sciences. 2015; 171:35965. [DOI:10.1016/j.sbspro.2015.01.133]

[13] Sadat SJ, Âli Mohammadi N, Âlamdari A. [Phenomenological study of family and social relationships of patients with Multiple Sclerosis (Persian)]. Journal of Mazandaran University of Medical Sciences. 2012;21(1):244-52. http://jmums.mazums.ac.ir/article1-969-en.html
[14] Heydari S, Salahshourian-fard A, Rafii F, Hoseini F. [Correlation of perceived social support from different supportive sources and the size of social network with quality of life in cancer patients (Persian)]. Iran Journal of Nursing. 2009; 22(61):8-18. http://ijn.iums. ac.ir/article-1-728-en.html

[15] Randolph DS, Andresen EM. Disability, gender, and unemployment relationships in the United States from the behavioral risk factor surveillance system. Disability \& Society. 2004; 19(4):403-14. [DOI:10.1 080/09687590410001689494]

[16] Simmons RD, Tribe KL, McDonald EA. Living with multiple sclerosis: Longitudinal changes in employment and the importance of symptom management. Journal of Neurology. 2010; 257(6):926-36. [DOI:10.1007/s00415-009-5441-7] [PMID]

[17] Moore P, Harding KE, Clarkson H, Pickersgill TP, Wardle M, Robertson NP. Demographic and clinical factors associated with changes in employment in Multiple Sclerosis. Multiple Sclerosis Journal. 2013, 19(12):1647-54. [DOI:10.1177/1352458513481396] [PMID]

[18] Dyck I, Jongbloed L. Women with multiple sclerosis and employment issues: A focus on social and institutional environments. Canadian Journal of Occupational Therapy. 2000; 67(5):337-46. [DOI:10. 1177/000841740006700506] [PMID]

[19] Ghafari Nasab E, Karimi K, Mosavat SE, Ghaseminejad MA. [The qualitative syudy of doctor-patient interaction patterns (Persian)]. Journal of Bioethics. 2017; 7(25):17-29. [DOI:10.22037/bioeth. v7i25.17368]

[20] Ghafari S, Fallahi Khoshknab M, Norozi K, Mohamadi E. [Exploring barriers of rehabilitation care in patients with Multiple Sclerosis: A qualitative study (Persian)]. Nursing and Midwifery Journal. 2014; 11(11):863-74. http://unmf.umsu.ac.ir/article-1-1696-en.html

[21] Ghanati E, Hadiyan M, Daghighi asli AR. [Economic expenditures of Multiple Sclerosis medications and feasibility of providing health insurance policies for medications (Persian)]. Journal of Health Administration. 2011; 14(45):37-54. http://jha.iums.ac.ir/article1-949-en.html

[22] Chiu C, Bishop M, Pionke J, Strauser D, Santens RL. Barriers to the accessibility and continuity of health-care services in people with Multiple Sclerosis: A literature review. International Journal of MS Care. 2017; 19(6):313-21. [DOI:10.7224/1537-2073.2016-016] [PMID] [PMCID]

[23] Raeisi R, Mohammadi MN, Almodaresi SA. [Determination of spatial and temporal distribution of MS Patients in Kerman (Persian)] Journal of Health Based Research. 2017; 3(3):217-26. http://hbrj. kmu.ac.ir/article-1-187-en.html

[24] Gromisch ES, Turner AP, Leipertz SL, Beauvais J, Haselkorn $\mathrm{JK}$. Who is not coming to clinic? A predictive model of excessive missed appointments in persons with Multiple Sclerosis. Multiple Sclerosis and Related Disorders. 2020; 38:101513. [DOI:10.1016/j. msard.2019.101513] [PMID]

[25] Khaleghpanah K. [Chronic illness as way of life: A study of the Multiple Sclerosis's lived realities (Persian)]. Sociological Review. 2016; 23(1):65-89.[DOI:10.22059/JSR.2016.58627]

[26] Ghodusi M, Heidari M, Sharifi Neyestanak N, Shahbazi S. [Correlation of perceived social support and some of the demographic factors in patients with Multiple Sclerosis (Persian)]. Journal of Health Promotion Management. 2013; 2(1):24-31. http://jhpm. ir/article-1-116-en.html 
[27] Movaghar S. [Effectiveness of mindfulness-based cognitive therapy to control dysfunctional thoughts in bad parent and runaway girls (Persian)]. Middle Eastern Journal of Disability Studies. 2017; 7(13):1-7. https://www.sid.ir/fa/journal/ViewPaper. aspx?ID $=306052$

[28] Kadkhodaee M, Azadfallah P, Farahani H. [A qualitative analysis of psychological well-being of women with regard to Iranian cultural structure (Persian)]. Journal of Developmental Psychology. 2018; 14(55):249-68. http://jip.azad.ac.ir/article_541253. htmllang=en

[29] Kirkpatrick Pinson DM, Ottens AJ, Fisher TA. Women coping successfully with Multiple Sclerosis and the precursors of change. Qualitative Health Research. 2009; 19(2):181-93. [DOI:10.1177/1049732308329465] [PMID] 